\title{
When Users Interfere with Protocols: Prospect Theory in Wireless Networks using Random Access and Data Pricing as an Example
}

\author{
Tianming Li and Narayan B. Mandayam, Fellow, IEEE
}

\begin{abstract}
Game theoretic models have found widespread use in the analysis and engineered system design of radio resource management algorithms for a wide variety of systems such as cellular, ad hoc and sensor networks. The fundamental principle behind such models and much of game theory has been the reliance on Expected Utility Theory (EUT). Motivated by the increasing amount of end-user control afforded in programmable radio devices, we envision a scenario where end-user actions essentially "interfere" with the underlying engineered system design. As an exemplary scenario, we consider in this paper wireless random access where players follow the precepts of Prospect Theory (PT), a theory developed by Kahneman and Tversky to explain real-life decision making that often deviates from the behavior expected under EUT. Specifically, we consider a game where selfish players adjust their transmission probabilities over a random access channel under throughput rewards, delay penalties and energy costs. By analyzing the Nash Equilibrium achieved, we prove under mild conditions that deviations from EUT results in degradation of system throughput while increasing delay and energy consumption. Finally, we consider a data pricing model and study the impact of enduser decision-making (i.e., players service choices) at the NE on wireless network performance.
\end{abstract}

Index Terms-Prospect theory, game theory, data pricing, wireless networks, random access.

\section{INTRODUCTION}

$\mathbf{S}$ INCE the early works in [1]-[5], game theory has emerged as a powerful tool for the analysis and design of radio resource management algorithms for wireless systems and networks. As detailed in recent surveys on game theoretical studies of various aspects of wireless communication networks [6] and [7], a great deal of meaningful insights have been gained into a wide range of problems and engineered system solutions have emerged for a variety of systems such as cellular, ad-hoc mesh, sensor and WiFi networks. All these works, including much of traditional game theory (going back to von Neumann and Morgenstern [8]) rely on the precept that users follow expected utility theory (EUT), where decisionmaking is guided strictly by accepted notions of utility, always

Manuscript received March 17, 2013; revised August 18 and December 11, 2013; accepted December 16, 2013. The associate editor coordinating the review of this paper and approving it for publication was T. Melodia.

This paper was presented in part as an invited paper at the 46th Annual Conference on Information Sciences and Systems(CISS), Princeton, NJ, March 21-23, 2012.

The authors are with WINLAB, ECE Department, Rutgers University, North Brunswick, NJ 08902 USA (e-mail: tmli.winlab@gmail.com, narayan@winlab.rutgers.edu).

Digital Object Identifier 10.1109/TWC.2013.021214.130472 rational and uninfluenced by real-life perceptions. This is a very sound assumption that governs the engineered system design of such systems when the actions of end-users do not interfere with such design. Moreover, there is ample evidence of the success of this approach as seen by the phenomenal growth of wireless system and network deployments along with their overarching applications and societal benefits.

On the other hand, the advent of easy to use, smart and programmable radio devices is resulting in the ability of endusers to control devices with a greater degree of freedom than ever. While current radio technologies and associated communication protocols are still for the most part agnostic to the decision- making of end-users, it is conceivable that in the future, users could make decisions that influence the underlying design of various algorithms and impact the performance of the overall system. These decisions could range from choices of access control to cooperation to selection of dynamic pricing plans. Other common examples of enduser actions include repeated refreshing of a browser under a delayed video stream or slowly loading web link. More sophisticated examples include modifying drivers of radio cards and associated protocols such as is increasingly becoming possible with the advent of programmable cognitive and smart radio devices. Even from a system perspective, the ever increasing capacity crunch faced by service providers is driving the migration of wireless data services in the future towards dynamic spectrum access and dynamic pricing based options, there by exposing the overall design of the network to the decisions of end-users based on their monetary perceptions of the value of the service. Further, there is ample evidence (anecdotal and otherwise) that decision making in real- life is often guided by perceptions that deviate from the precepts of EUT.

Motivated by these emerging wireless networking scenarios, we turn to Prospect Theory (PT) [9], a Nobel prize winning theory developed by Kahneman and Tversky that explains reallife decision- making and its deviations from EUT behavior. While the main ideas and models behind early PT were developed based on responses/decisions of players involving monetary transactions (prospects), the behavioral deviations from EUT are general enough that they have widespread application in many areas [10]-[13]. We believe that understanding the role of PT in wireless systems and networks, that are increasingly becoming user-centric is important. It is often recognized that a measurement of user satisfaction must be included in the assessment of the efficiency of the 
network as a whole [14]. It has also been revealed that service repurchase intention among mobile Internet users was significantly positively related to "experienced value" and "satisfaction" [15]. While these findings highlight the importance of emphasizing user-experience when defining and assessing quality of service, traditionally, improvement in network service has followed a bottom-up approach, assuming that optimization of performance at the engineering design level will translate directly into an improved user experience.

As a first step in this direction, we consider an exemplary radio resource management problem where users follow PT and compare and contrast it to the case when users follow EUT. Specifically, we consider a random access game where selfish players adjust their transmission probabilities over a collision channel according to rewards received for successful transmission but also incur energy and delay costs. In the initial work [16], we only considered a 2-player homogeneous wireless random access game with $0 / 1$ collision channel. In this paper, we significantly extend the work by studying and comparing both 2-player homogeneous and heterogeneous games where general random access channel model can be applied, i.e., the packet reception probability can range within $[0,1]$. Furthermore, we also extend the investigation to a $\mathrm{N}$ player homogeneous game. Our extended results prove the correctness of our findings in [16] in a much more general fashion, i.e., the deviation from EUT results in degradation of system throughput, increased delay and energy consumption. Moreover, we study the impact of end users' deviation from EUT in a more practical scenario, i.e., data pricing. Under the basic setting as in the wireless random access network, a 2-tier data pricing network is studied under two different data pricing schemes( UBP and TBP ).

While the random access scenario considered here does not exactly reflect the time-scale or granularity of end-user decision making, it never the less serves as a useful illustration to open up this new line of investigation. There is a gap between the random access model used here and practical role of PT in the real world. However, we believe this exemplary model can get rid of the unnecessary complexities of real wireless systems at the very initial stage of this new research and thus help us identify the possible impact of end-user behaviors on wireless systems in a clear fashion. Moreover, this simple wireless random access model actually captures the essence of some real-life wireless communication scenarios. Notable examples of end-user decision making as it relates to transmission probabilities are: the decision to access cellular data services when the network may already be congested; the decision to utilize a Wi-Fi service at an airport depending on the usage fee and relative importance of immediate data communications; and the decision to utilize high speed data services even though the battery levels may be very low. The random access model used in this paper may not exactly describe the above scenarios, however, it is actually a reasonable abstraction of the transmit choices made by the end user since it focuses on the probability of a user's data transmission.

The rest of the paper is organized as follows. An extensive related work review is provided in section II. In section III, a brief introduction to Prospect Theory is provided. Wireless random access games are formulated in section IV among selfish players under both the EUT and PT models. In section $\mathrm{V}$, the impact of any player's deviation from EUT on the individual player's and system performance is numerically and analytically studied in a 2-player heterogeneous wireless random access game. The scenario where both players follow either only PT or only EUT is further studied as 2-player homogeneous PT game and EUT game, respectively in section VI. In section VII, a N-player homogeneous game where all the users follow either only PT or only EUT is discussed. We build on the work in the previous sections and use PT in section VIII to study a 2-tier data pricing network. The performance variations due to the deviation of end-user decision making from EUT are observed and analytically studied. We conclude in section IX. A list of important notations is provided in Table II for the readers' convenience.

\section{RELATED WORK}

As mentioned in section II, the advances in smart and programmable radio devices have provided the end users with a greater degree of control [17]-[19]. As a result, the understanding of end users' real-life decisions are becoming of paramount importance in designing and managing current and future wireless networks. Prospect Theory (PT) is well known to be able to explain real-life decision making and thus becomes a candidate tool to address this need. Though historically, PT has been developed based on monetary transactions, it has been successfully applied to various fields as mentioned in the above section, such as [10]-[13]. Game theory has also been a powerful tool to describe the interactions among end users in wireless networks as some exemplary works can be found in [1]-[5], [20], [21]. The game theoretical approach heavily relies on the precept that users follow Expected Utility Theory (EUT). In [22], the authors have provided basic characterizations of non-cooperative games where players may follow PT for decision making.

As indicated in section $\mathrm{I}$, research is needed to identify how resource allocation mechanisms impact the value of that resource to users and conversely, how end-user actions impact resource allocation. The end users' subjective perception over the received service and their behaviors have more and more significant influence on the design, operation and performance of communication networks and systems [23]. The authors in [24] explore the possible usage of psychology in the field of autonomous networks and systems. They claim that the end users' behaviors and preferences can have direct impact over the operation and design of the such systems. They used PT as a possible candidate to model end users' preferences. In [25], the authors identify the importance of the end users' behaviors and preferences and proposed a "local community networking system with ad hoc networking technology". Moreover, they used Prospect Theory to model the end users' behaviors in such a relaying network environment.

Though the above works have conceptually mentioned the need for Prospect Theory in communication networks and systems, a more in-depth technical and quantitative study on this topic is needed. Therefore, we select an exemplary wireless random access model as a starting point to identify the impact of end users' decisions on the wireless networks 
with the help of PT. The work in [2] is one of first works that applies game theory to wireless random access and power control. Specifically, the authors applied game theory to a simplified Aloha system. In [26], a more detailed game-theoretical study was provided on an Aloha system. The authors in [27] have investigated the probabilities of retransmission in a distributed manner under both cooperative model and noncooperative game model in a slotted Aloha system. In [28], the authors have performed an extensive study on the end users' transmission probabilities at the equilibrium of a random access network considering end users' throughput rewards, energy costs and delay penalties. Data pricing in wireless networks has been extensively studied under EUT models, examples of which can be found in [5], [29]-[32] and therein.

\section{BACKground: Prospect Theory}

Expected Utility Theory (EUT) [8] has been a fundamental part of modern economics. It provides an approach to evaluate a prospect $L$ [9], i.e., a contract that will yield $M$ different outcomes $o_{i}, i=1, \ldots, M$ and each outcome occurs with probability $p_{i}, \forall i=1, . ., M$ where $\sum_{i=1, \ldots, M} p_{i}=1$. EUT determines that the prospect is evaluated as

$$
u^{E U T}(L)=\sum_{i=1, \ldots, M} p_{i} v^{E U T}\left(o_{i}\right),
$$

i.e., the expected value of all possible outcomes. $v^{E U T}(\cdot)$ is a value function of the outcomes and it is often assumed to be concave in EUT. However, in Prospect Theory (PT) [9] that was postulated by Kahneman and Tversky, extensive experiments and measurements suggest that in decision-making in real life, the prospect $L$ is evaluated as

$$
u^{P T}(L)=\sum_{i=1, \ldots, M} w\left(p_{i}\right) v^{P T}\left(o_{i}\right) .
$$

This valuation is significantly different from EUT in the following two ways.

(1) Probability Weighting Effect: It is revealed in PT that people use their subjective probabilities $w\left(p_{i}\right)$ rather than objective probabilities $p_{i}$ to weigh the values of possible outcomes. Moreover, people tend to over-weigh low probability outcomes and under-weigh moderate and high probability outcomes. Based on the experimental results, an original form for the probability weighting function was proposed in [33]. While there have been several efforts to identify appropriate probability weighting functions, in this paper, we will use the one identified by Prelec [34] that captures the over-weighting and underweighting of probabilistic outcomes as follows (see Fig. 1):

$$
w(p)=\exp \left(-(-\ln p)^{\alpha}\right), 0<\alpha \leq 1,
$$

where $\alpha$ is the parameter which reveals how a person's subjective evaluation distorts the objective probability and a smaller $\alpha$ describes a more curved probability weighting function. From Fig. 1, we can see that the probability weighting function has several features [34]: (1) It is asymmetrically reflected at a point, i.e., $1 / e$, where $w(1 / e)=1 / e ;(2)$ It is concave if $0 \leq p<1 / e$

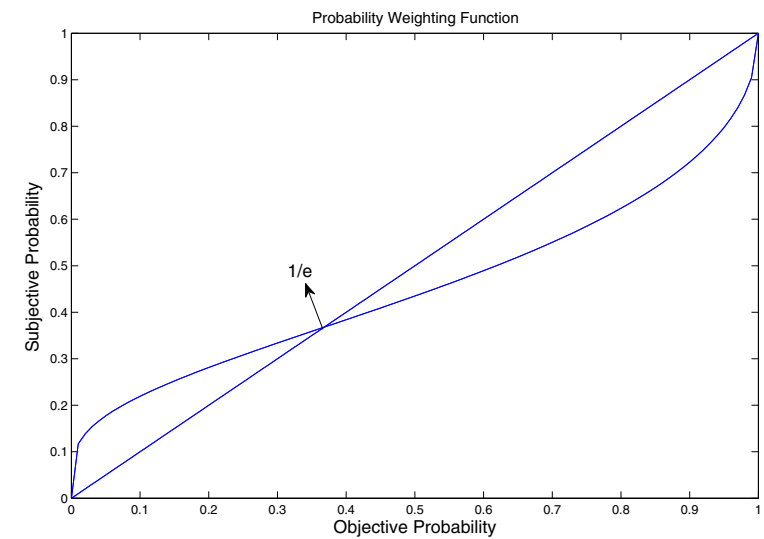

Fig. 1. Probability Weighting Function. The curve shows the probability weighting effects when $\alpha=0.5$. The straight line represents the objective probability.

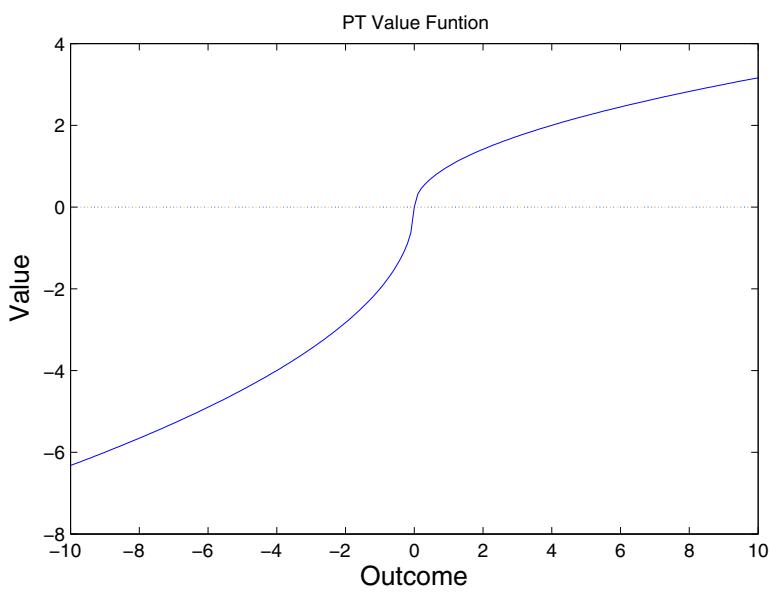

Fig. 2. Framing Effects in Prospect Theory. A generic value function is shown about reference point 0 .

and convex if $1 / e \leq p \leq 1$; (3) $w(p)>p$ if $0 \leq p<1 / e$ and $w(p) \leq p$ if $1 / e \leq p \leq 1$.

(2) Framing Effect: PT [9] states that in decision-making in real life, the value of an outcome is determined by considering the relative gains or losses regarding a reference point. PT also proposes that the value function should be a concave function of gains and a convex function of losses with the convex part usually having a steeper slope. In other words [9], "losses looms larger than gains." The framing effects can be demonstrated in Fig. 2.

It has been consistently observed that EUT fails to interpret people's real-life decisions that could be well explained by PT, e.g., the famous Allais' paradox. In [9], the authors provided a variation of the Allais' paradox as in Table I. There were two problems in the experiment and for each problem, the respondents were asked to choose between two prospects (A or B). For example, in Table I, the respondent had two prospects in problem 1 . If she chose A, she would win 2500 dollars with probability 0.33 or 2400 dollars with probability 0.66 or nothing with probability 0.01 . If she chose B, she would win 2400 dollars for sure. In [9], it 
TABLE I

AN EXAMPLE OF EUT ViOLATION

\begin{tabular}{|c|c|c|}
\hline Problem & $A$ & $B$ \\
\hline 1 & Prospect & A \\
& $\$ 2500$ with probability 0.33 & $\$ 2400$ with certainty \\
& $\$ 2400$ with probability 0.66 & \\
& $\$ 0$ with probability 0.01 & \\
\hline 2 & $\$ 2500$ with probability 0.33 & $\$ 2400$ with probability 0.34 \\
& \$0 with probability 0.67 & \$0 with probability 0.66 \\
\hline
\end{tabular}

was found that a majority of the respondents (61 per cent) chose B for problem 1 and A for problem 2. According to EUT, a respondent would evaluate a prospect, e.g., problem $1 \mathrm{~A}$, as the expectation of all the prospect's outcomes, e.g., $0.33 v^{E U T}(2500)+0.66 v^{E U T}(2400)+0.01 v^{E U T}(0)$. Thus, a preference of $1 \mathrm{~B}$ over $1 \mathrm{~A}$ implies $0.33 v^{E U T}(2500)+$ $0.66 v^{E U T}(2400)+0.01 v^{E U T}(0)<v^{E U T}(2400)$ that is equivalent to $0.34 v^{E U T}(2400)>0.33 v^{E U T}(2500)$. Meanwhile, the choice of $2 \mathrm{~A}$ over $2 \mathrm{~B}$ implies $0.34 v^{E U T}(2400)<$ $0.33 v^{E U T}(2500)$. These two results produce a paradox. This observation as well as numerous other ones are used in [13] to illustrate the situations where EUT fails to accurately describe people's real-life decisions. However, PT can successfully explain the decisions the respondents made in the above experiment. Given zero is the reference point, $\alpha=0.5$ and $v^{P T}(\cdot)$ is linear, it can be easily identified that $w(0.33) v^{P T}(2500)+$ $w(0.66) v^{P T}(2400)+w(0.01) v^{P T}(0)<w(1) v^{P T}(2400)$ and $w(0.34) v^{P T}(2400)<w(0.33) v^{P T}(2500)$ are established simultaneously. Unlike the framing effect shown in Fig. 2, in this paper we apply PT by assuming a linear value function towards the outcomes of communication activities, i.e., $v^{P T}(x)=a x$ where $a \geq 0$ is a constant. Note that, $x$ is the gain or loss relative to fixed reference point zero, i.e., values are framed about the reference point zero.

\section{A Wireless Random Access Game}

In this paper, we consider a time slotted wireless random access network with $N=|\mathcal{N}|$ selfish players accessing a single access point. Each player is assumed to have a saturated packet queue and able to transmit one packet in a time slot [28]. Given any time slot, there is a subset $\mathcal{J} \subseteq \mathcal{N}$ of players that transmit packets to the base station simultaneously while players in $\mathcal{J}^{c} \subseteq \mathcal{N}$ decide to wait. For player $i \in \mathcal{J}$, let $p_{i \mid \mathcal{J}}$ be the probability that the base station captures her packet. Note that, player $i$ 's transmission probability is denoted as $p_{i}$. For a successful packet delivery, a player obtains a unit reward $c_{i} \geq 0$ while incurring a unit energy $\operatorname{cost} e_{i} \geq 0$. However, if a player $i \in \mathcal{J}$ fails to deliver her packet to the base station, she incurs a unit energy cost $e_{i} \geq 0$ and a unit delay penalty $d_{i} \geq 0$. If she decides to not transmit (wait), i.e., $i \in \mathcal{J}^{c}$, she also incurs a unit delay penalty $d_{i} \geq 0$.

All the players have the same pure strategy set, i.e., $A_{i}=$ $\{t, n t\}, i \in \mathcal{N}$, where the strategy $t$ denotes transmission and $n t$ denotes wait (no transmission). The pure strategy profile of all $N$ players in a time slot can be represented as a vector $\mathbf{a}=\left[a_{i}\right]_{\forall i \in \mathcal{N}} \in \mathbf{A}$ where $\mathbf{A}=A_{1} \times A_{2} \times \ldots \times A_{N}$ is the set of all possible pure strategy profiles. For any given $\mathbf{a} \in \mathbf{A}$, let $\mathcal{J}(\mathbf{a}) \subseteq \mathcal{N}$ denote the set of players who transmit. Each transmission generates two possible outcomes with probabilities for each player $i \in \mathcal{J}(\mathbf{a})$, i.e., a successful packet delivery with probability $p_{i \mid \mathcal{J}(\mathbf{a})} \in[0,1]$ or a packet delivery

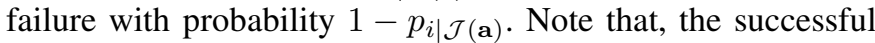
packet delivery probability $p_{i \mid \mathcal{J}(\mathbf{a})}$ is actually determined by the underlying channel model, e.g., path loss, AWGN and rayleigh. The exact channel model adopted is not crucial in this model and thus $p_{i \mid \mathcal{J}(\mathbf{a})}$ is assumed to be available for each player. If the player $i$ does not transmit, i.e., $i \in \mathcal{J}(\mathbf{a})^{c}$, she gets a delay penalty $d_{i}$. Thus, if we assume a linear value function, then a player evaluates the possible outcomes associated with an arbitrary pure strategy profile as follows:

$v_{i \mid \mathbf{a}}= \begin{cases}p_{i \mid \mathcal{J}(\mathbf{a})}\left(c_{i}-e_{i}\right)+\left(1-p_{i \mid \mathcal{J}(\mathbf{a})}\right)\left(-e_{i}-d_{i}\right) & a_{i}=t \\ -d_{i} & a_{i}=n t .\end{cases}$

Under this setting, we will consider random access games with 2 types of players: (a) players who follow the precepts of EUT and (b) players who follow the precepts of PT. We will refer to these players as EUT players and PT players, respectively. Further, depending on the player composition, two types of wireless random access games are possible: (1) homogeneous game and (2) heterogeneous game. A homogeneous game consists of either only EUT players or only PT players. The heterogeneous games must simultaneously have both PT and EUT players, i.e., some players are PT players and others are EUT players. Furthermore, under linear value function and framing about 0 , the PT and EUT players have identical value functions over a pure strategy profile, i.e., $v_{i \mid \mathbf{a}}^{E U T}=v_{i \mid \mathbf{a}}^{P T}=$ $v_{i \mid \mathbf{a}}$. We assume each player may adopt a mixed strategy, i.e., player $i$ will transmit a packet in a time slot with probability $p_{i} \in[0,1]$ and wait with probability $1-p_{i}$. A mixed strategy vector of all players can be represented as $\mathbf{p}=\left[p_{1}, p_{2}, \ldots, p_{N}\right]$ where $p_{i}$ is the $i$-th player's transmission probability. An EUT player's utility can be represented as,

$$
u_{i}^{E U T}(\mathbf{p})=\sum_{\mathbf{a} \in \mathbf{A}}\left(\prod_{j \in \mathcal{J}(\mathbf{a})} p_{j} \prod_{k \notin \mathcal{J}(\mathbf{a})}\left(1-p_{k}\right) v_{i \mid \mathbf{a}}\right),
$$

where $\mathcal{J}(\mathbf{a})$ is the set of players who transmit in the time slot, i.e., $a_{j}=t, \forall j \in \mathcal{J}(\mathbf{a})$. Note that, the probability that a specific pure strategy profile $\mathbf{a}$ is chosen is $\prod_{j \in \mathcal{J}(\mathbf{a})} p_{j} \prod_{k \notin \mathcal{J}(\mathbf{a})}\left(1-p_{k}\right)$ and $v_{i \mid \mathbf{a}}$ is the corresponding value for player $i$ as defined in (4).

A PT player knows that the strategy profile a will occur with probability $\prod_{j \in \mathcal{J}(\mathbf{a})} p_{j} \prod_{k \notin \mathcal{J}(\mathbf{a})}\left(1-p_{k}\right)$. However, she cannot objectively evaluate this probability and this makes her utility function fundamentally different from that of an EUT player as discussed in section II. A PT player's subjective utility can be formally defined as:

$$
u_{i}^{P T}(\mathbf{p})=\sum_{\mathbf{a}_{1} \in \mathbf{A}, a_{1 i}=t} S P\left(\mathbf{a}_{1}\right) v_{i \mid \mathbf{a}_{1}}+\left(1-p_{i}\right)\left(-d_{i}\right) .
$$

Note in the above, the player $i$ subjectively determines that $\mathbf{a}_{1}$ will be chosen with probability $S P\left(\mathbf{a}_{1}\right)=$ $p_{i} w_{i}\left(\prod_{j \in \mathcal{J}\left(\mathbf{a}_{1}\right) \backslash\{i\}} p_{j} \prod_{k \in \mathcal{J}^{c}\left(\mathbf{a}_{1}\right)}\left(1-p_{k}\right)\right)$, where $\mathbf{a}_{1} \in \mathbf{A}$ denotes the pure strategy profiles where the $i$-th player transmits, i.e., $a_{1 i}=t$, and $w_{i}(\cdot)$ is the $i$-th player's probability weighting function. Similarly, she weighs outcomes of $\mathbf{a}_{2}$ with $S P\left(\mathbf{a}_{2}\right)=\left(1-p_{i}\right) w_{i}\left(\prod_{j \in \mathcal{J}\left(\mathbf{a}_{2}\right)} p_{j} \prod_{k \in \mathcal{J}^{c}\left(\mathbf{a}_{2}\right) \backslash\{i\}}\left(1-p_{k}\right)\right)$ 
where $\mathbf{a}_{2} \in \mathbf{A}$ denotes the pure strategy profiles where she chooses to wait, i.e., $a_{2 i}=n t$. Note that, we assume a PT player only nonlinearly transforms other players' strategy probabilities rather than her own since a person is able to choose her own strategy probability objectively.

For the $i$-th player, she plays the random access game by solving an optimization problem as follows:

$$
\max _{p_{i}} u_{i}\left(p_{i}, p_{-i}\right),
$$

where $u_{i}(\cdot)$ can be either EUT utility (5) or PT utility (6) and $p_{-i}$ represents the collection of all other players' transmission probabilities. A list of important notations is list in Table II.

\section{A 2-Player Heterogeneous Wireless Random ACCESS GAME}

In our earlier work [16], a 2-player homogeneous wireless random access game with linear value function was studied using a simple $0-1$ random collision channel model for random access. Here, we investigate a 2-player heterogeneous wireless random access game with linear value function for a general random access channel. We extensively study the differences between a 2-player homogeneous EUT game and a 2-player heterogeneous game. In the following, we use $i$ to denote the player we refer to and $j$ to denote her opponent. According to (5) and (6), the utility functions of an EUT and a PT player can be specified as follows, for $i=1,2$ :

$$
\begin{aligned}
u_{i}^{E U T}(\mathbf{p})= & p_{i} p_{j} v_{i \mid\{t, t\}}+p_{i}\left(1-p_{j}\right) v_{i \mid\{t, n t\}} \\
& +\left(1-p_{i}\right)\left(-d_{i}\right) \\
u_{i}^{P T}(\mathbf{p})= & p_{i} w_{i}\left(p_{j}\right) v_{i \mid\{t, t\}}+p_{i} w_{i}\left(1-p_{j}\right) v_{i \mid\{t, n t\}} \\
& +\left(1-p_{i}\right)\left(-d_{i}\right)
\end{aligned}
$$

where $v_{i \mid\{t, t\}}=p_{i \mid\{i, j\}}\left(c_{i}-e_{i}\right)+\left(1-p_{i \mid\{i, j\}}\right)\left(-e_{i}-d_{i}\right), i=$ 1,2 is the value the $i$-th player assigns for the pure strategy $\{t, t\}$, i.e., both players transmit. $v_{i \mid\{t, n t\}}=p_{i \mid\{i\}}\left(c_{i}-e_{i}\right)+$ $\left(1-p_{i \mid\{i\}}\right)\left(-e_{i}-d_{i}\right), i=1,2$ denotes the value the $i$-th player assigns for the pure strategy profile $\{t, n t\}$, i.e., only the $i$-th player transmits. Note that, the first element in a pure strategy profile is always the referred player's strategy. $p_{i \mid\{i, j\}}$ and $p_{i \mid\{i\}}$ denote the $i$-th player's successful packet delivery probabilities given that her opponent player $j$ also transmits or not, respectively. $p_{i}$ and $p_{j}$ represent the transmission probabilities of the $i$-th player and her opponent, respectively. Under the game setting, players' communication performance are compared for three metrics, namely average throughput, average energy and average delay. They can be calculated as follows, for $i=1,2$ :

$$
\begin{aligned}
& T_{i}(\mathbf{p})=p_{i} p_{j} p_{i \mid\{i, j\}}+p_{i}\left(1-p_{j}\right) p_{i \mid\{i\}} \\
& E_{i}(\mathbf{p})=p_{i} \\
& D_{i}(\mathbf{p})=p_{i} p_{j}\left(1-p_{i \mid\{i, j\}}\right)+p_{i}\left(1-p_{j}\right)\left(1-p_{i \mid\{i\}}\right)+\left(1-p_{i}\right)
\end{aligned}
$$

\section{A. The Existence and Uniqueness of Mixed NEs of a Hetero- geneous Game and a Homogeneous EUT game}

The performance of players and system in (10) - (12) can only be meaningfully evaluated at Nash Equilibrium (NE)
TABLE II

NOTATIONS REFERENCE TABLE

\begin{tabular}{|c|c|}
\hline Notations & Interpretations \\
\hline $\mathcal{N}$ & Set of players \\
\hline $\mathbf{a}$ & A pure strategy profile vector of all players \\
\hline $\mathbf{p}$ & A mixed strategy profile vector of all players, i.e. $\left\{p_{i}\right\}$ \\
\hline $\mathcal{J}\{\mathbf{a}\}$ & Set of players choosing transmission given a \\
\hline$v_{i \mid \mathbf{a}}$ & The value $i$-th player obtains given a \\
\hline$p_{i}$ & The $i$-th player's transmission probability \\
\hline$p_{i \mid \mathcal{J}(\mathbf{a})}$ & The $i$-th player's successful package delivery probability given $\mathcal{J}(\mathbf{a})$ \\
\hline$w_{i}(\cdot)$ & The $i$-th player's probability weighting function \\
\hline$u_{i}^{E U T}(\cdot)$ & The $i$-th EUT player's utility \\
\hline$u_{i}^{P T}(\cdot)$ & The $i$-th PT player's utility \\
\hline$v_{i \mid\{t, n t\}}$ & The $i$-th player's value when she transmits and her opponent stays quite \\
\hline$p_{i \mid\{i, j\}}$ & The $i$-th player's package reception probability when both players transmit \\
\hline
\end{tabular}

TABLE III

Players' VALUES TOWARDS PURE STRATEGY PROFILES

\begin{tabular}{|c|c|c|}
\hline Player 1 Player 2 & $t$ & $n t$ \\
\hline$t$ & $\left(v_{1 \mid\{t, t\}}, v_{2 \mid\{t, t\}}\right)$ & $\left(v_{1 \mid\{t, n t\}},-d_{2}\right)$ \\
\hline$n t$ & $\left(-d_{1}, v_{2 \mid\{t, n t\}}\right)$ & $\left(-d_{1},-d_{2}\right)$ \\
\hline
\end{tabular}

[35]. In the following, the mixed NE characteristics of a heterogeneous game and a homogeneous EUT game are studied. The players' utilities associated with pure strategy profiles can be compactly described in table III. Note that, if the strategy profile $\{t, t\}$ where both players transmit is more preferred by player $i$ than the strategy where player $i$ does not transmit, i.e., $v_{i \mid\{t, t\}} \geq-d_{i}, i=1,2$, it is also true that $v_{i \mid\{t, n t\}}>-d_{i}$ since the profile $\{t, n t\}$ generates less interference to player $i$ 's transmission and thus more preferred than the strategy profile $\{t, t\}$. As a result, player $i$ will always transmit, i.e., $p_{i}=1$. Similarly, if $-d_{i} \geq v_{i \mid\{t, n t\}}>v_{i \mid\{t, t\}}, i=1,2$, player $i$ will always not transmit by adopting $p_{i}=0$. In the following, we exclude these two trivial scenarios by assuming for $i=1,2, v_{i \mid\{t, t\}}<-d_{i}$ and $v_{i \mid\{t, n t\}}>-d_{i}$. Furthermore, in the following, no further discussion on the resulting two trivial pure NEs, i.e., $\{t, n t\},\{n t, t\}$, will be made either.

Theorem 1. There exists a unique mixed $N E\left[p_{1}^{H}, p_{2}^{H}\right]$ for a heterogeneous game given $v_{i \mid\{t, t\}}<-d_{i}$ and $v_{i \mid\{t, n t\}}>0$ for $i=1,2$.

Proof: Without loss of generality, we assume player 1 is a PT player with utility function (9) and player 2 is an EUT player with utility function (8). In this game, each player, e.g., player $i, i=1,2$, will choose a probability for transmission, e.g., $p_{i} \in \Delta\left(A_{i}\right)=[0,1]$ for $t$ and thus $1-p_{i}$ for $n t$. It can be easily seen that the interval $\Delta\left(A_{i}\right)$ is a nonempty compact convex subset of a Euclidian space for $i=1,2$. Further, the PT player's utility function in (9) is linear in $p_{1} \in \Delta\left(A_{1}\right)$. Given a fixed $p_{2}$ (i.e., the EUT player's mixed strategy), we pick any feasible $b_{1}, f_{1}, g_{1} \in$ $\Delta\left(A_{1}\right)$ and $u_{1}^{P T}\left(b_{1}, p_{2}\right) \geq \max \left\{u_{1}^{P T}\left(f_{1}, p_{2}\right), u_{1}^{P T}\left(g_{1}, p_{2}\right)\right\}$. Then, $u_{1}^{P T}\left(\lambda f_{1}+(1-\lambda) g_{1}, p_{2}\right)=\lambda u_{1}^{P T}\left(f_{1}, p_{2}\right)+(1-$ 入) $u_{1}^{P T}\left(g_{1}, p_{2}\right)<u_{1}^{P T}\left(b_{1}, p_{2}\right), \forall \lambda \in[0,1]$. Hence, the subset of the PT player's mixed strategies which yield less utility than an arbitrary feasible mixed strategy $b_{1} \in \Delta\left(A_{1}\right)$ is convex and we have shown the PT player's utility function is quasiconcave in $\Delta\left(A_{1}\right)$. Similarly, it can be easily shown that the EUT player's utility function $u_{2}^{E U T}\left(p_{1}, p_{2}\right)$ is also quasiconcave in $\Delta\left(A_{2}\right)$. According to the Proposition 20.3 in [35], at least one mixed $\mathrm{NE}$ exists in the heterogeneous game. 
At the mixed NE $\mathbf{p}^{*}=\left[p_{1}^{*}, p_{2}^{*}\right]$, by definition, the PT player should always obtain the identical utility by playing either of the pure strategies, i.e., $u_{1}^{P T}\left(1, p_{2}^{*}\right)=u_{1}^{P T}\left(0, p_{2}^{*}\right)$. Note that, $u_{1}^{P T}\left(1, p_{2}\right)=w_{1}\left(p_{2}\right) v_{1 \mid\{t, t\}}+w_{1}\left(1-p_{2}\right) v_{1 \mid\{t, n t\}}$ and $u_{1}^{P T}\left(0, p_{2}\right)=-d_{1}$. If $v_{1 \mid\{t, t\}}<0$ and $v_{1 \mid\{t, n t\}}>0$, the PT player assigns a negative value to a collision and a positive value to an interference-free transmission. Then, it follows that

$$
\begin{aligned}
& \frac{\partial u_{1}^{P T}\left(1, p_{2}\right)}{\partial p_{2}}=\frac{\alpha w_{1}\left(p_{2}\right)\left(-\ln \left(p_{2}\right)\right)^{\alpha-1}}{p_{2}} v_{1 \mid\{t, t\}} \\
& \quad+\frac{\alpha w_{1}\left(1-p_{2}\right)\left(-\ln \left(1-p_{2}\right)\right)^{\alpha-1}}{p_{2}-1} v_{1 \mid\{t, n t\}}<0 .
\end{aligned}
$$

Therefore, $u_{1}^{P T}\left(1, p_{2}\right)$ is a strictly decreasing function of $p_{2} \in$ $[0,1]$. The points where the function $u_{1}^{P T}\left(1, p_{2}\right)$ intersects with the horizontal line $u_{1}^{P T}\left(0, p_{2}\right)=-d_{1}$ are the solutions to the equation $u_{1}^{P T}\left(1, p_{2}\right)=u_{1}^{P T}\left(0, p_{2}\right)$ and thus the EUT player's mixed strategies at the mixed NE. Since $u_{1}^{P T}\left(1, p_{2}\right)$ has been shown as a strictly decreasing function of $p_{2} \in[0,1]$, the intersection point is unique and therefore the EUT player's mixed strategy (transmission probability) $p_{2}^{\mathrm{H}}$ at the mixed NE is also unique. Meanwhile, the EUT player's utility when she always transmits, i.e., $u_{2}^{E U T}\left(p_{1}, 1\right)$, can be similarly shown as a strictly decreasing function of $p_{1} \in[0,1]$. Hence, the PT player's mixed strategy $p_{1}^{\mathrm{H}}$ at the mixed $\mathrm{NE}$ is also unique. Furthermore, as mentioned earlier, $v_{i \mid\{t, t\}}<-d_{i}, i=1,2$ and $v_{i \mid\{t, n t\}}>0>-d_{i}, i=1,2$ ensure that neither of the players would choose a pure strategy. Thus, a unique mixed $\mathrm{NE}\left[p_{1}^{\mathrm{H}}, p_{2}^{\mathrm{H}}\right]$ exists for the 2-player heterogeneous game.

Note that, the conditions in Theorem 1 are mild and intuitive in that they reflect a communications scenario where a "positive" value $\left(v_{i \mid\{t, n t\}}>0, i=1,2\right)$ results when only player $i$ transmits and a "negative" value $\left(v_{i \mid\{t, t\}}<-d_{i}, i=1,2\right)$ results when the 2 players collide. Further, this negative value is worse than when the player $i$ does not transmit.

Corollary 2. There exists a unique mixed $N E\left[p_{1}^{E U T}, p_{2}^{E U T}\right]$ for the homogeneous EUT game given $v_{i \mid\{t, t\}}<-d_{i}$ and $v_{i \mid\{t, n t\}}>0$ for $i=1,2$.

Proof: The existence and uniqueness of the mixed NE for the homogeneous EUT game can be established in the same fashion as in the heterogeneous game.

Due to the analytical intractability of the probability weighting function in equation (3), the mixed NE involving PT players cannot be derived in closed form even for a 2-player scenario. For example, in a 2-player homogeneous game, the players' transmission probabilities $\left[p_{1}, p_{2}\right]$ at the mixed NE can be derived by solving the following nonlinear equations:

$$
\begin{gathered}
\exp \left(-\left(-\ln p_{1}\right)^{\alpha}\right) v_{2 \mid\{t, t\}}+\exp \left(-\left(-\ln \left(1-p_{1}\right)\right)^{\alpha}\right) v_{2 \mid\{t, n t\}}=-d_{2} \\
\exp \left(-\left(-\ln p_{2}\right)^{\alpha}\right) v_{1 \mid\{t, t\}}+\exp \left(-\left(-\ln \left(1-p_{2}\right)\right)^{\alpha}\right) v_{1 \mid\{t, n t\}}=-d_{1} .
\end{gathered}
$$

Even though the above 2 equations are independent, owing to the analytical intractability in arriving at closed form solution, we use the numerical solvers in MATLAB to find the transmission probabilities at the mixed NE in the rest of the paper. In spite of the analytical intractability, we will prove many intuitive properties of the mixed NE as will be seen in the remainder of the paper.

\section{B. A Heterogeneous Game: Consequence of Deviation from EUT}

A heterogeneous game consisting of one PT player and one EUT player is considered and we will highlight the consequences on the performance compared to a 2-player homogeneous EUT game. The heterogeneous game can be considered as a deviation from the homogeneous game as one of the EUT players is replaced by a PT player who differs from the EUT players in the homogeneous game only in the probability weighting index $\alpha$. We specifically study three issues: (a) the impact on the performance of the EUT player in the heterogeneous game, (b) the impact on the performance of the whole system, and (c) the performance difference between the PT and EUT players in the heterogeneous game. We consider the following 3 scenarios for comparison: (1) Both players are EUT (EUT game), (2) One EUT and one PT player with $\alpha=0.8$, and (3) One EUT and one PT player with $\alpha=$ 0.5 . For both the heterogeneous game and the homogeneous EUT game, two players randomly access the channel with $p_{i \mid\{i\}}=p_{j \mid\{j\}}=0.98, p_{i \mid\{i, j\}}=p_{j \mid\{i, j\}}=0.05, i=1,2$. We begin with a special case where there is no delay penalty for each player as $c_{1}=c_{2}=c, e_{1}=e_{2}=e=2, d_{1}=d_{2}=$ $d=0$. The system performances are studied and compared as a function of $c / e$. Due to the symmetry here, the EUT players in the homogeneous EUT game have the identical strategy and performance at the mixed NE. We observed in Fig. 3(a) that with the introduction of the PT player, the EUT player transmits more aggressively in the heterogeneous game than the EUT players in the homogeneous EUT game. Note that, the PT player in the heterogeneous game under two different scenarios chooses the same transmission probability at the mixed NE since her opponent's (the EUT player) utility function stays unchanged. Within the heterogenous game, the EUT player is more aggressive in transmission than the PT player. Both the trends are more obvious as the PT player deviates more from EUT (smaller $\alpha$ ). Further, both the players' transmissions under the 3 scenarios become more probable as the unit throughput reward increases and finally converge to transmission with probability 1 when $v_{i \mid\{t, t\}}=-d=0, i=$ $1,2(c / e=20)$. Note that, all the curves do not increase to 1 until $c / e=20$ though in the figure, they are seen very close to it before $c / e=20$.

In Fig. 3(b), it can be seen that the introduction of the PT player actually causes the EUT player in the heterogeneous game consume more average energy than the EUT players in the homogeneous EUT game. Meanwhile, the PT player's consumed energy stays unchanged as explained in Fig. 3(a). As a result, the players in the heterogeneous game consume more sum energy in average than those in the homogeneous EUT game. Moreover, a more deviated PT player causes an increased sum average energy consumption.

Figure 4(a) shows that the introduction of the PT player actually increases the EUT player's average throughput compared to the EUT players in the homogeneous EUT game. However, the PT player herself suffers an obvious average throughput degradation. Moreover, within the heterogenous game, the PT player achieves an obviously less average throughput than the EUT player. Further, as the PT player 


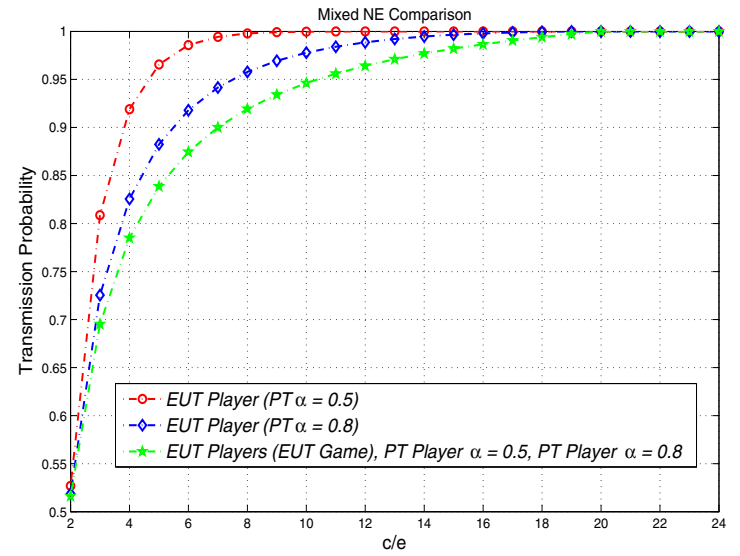

(a) Transmission probability of each player at mixed NE

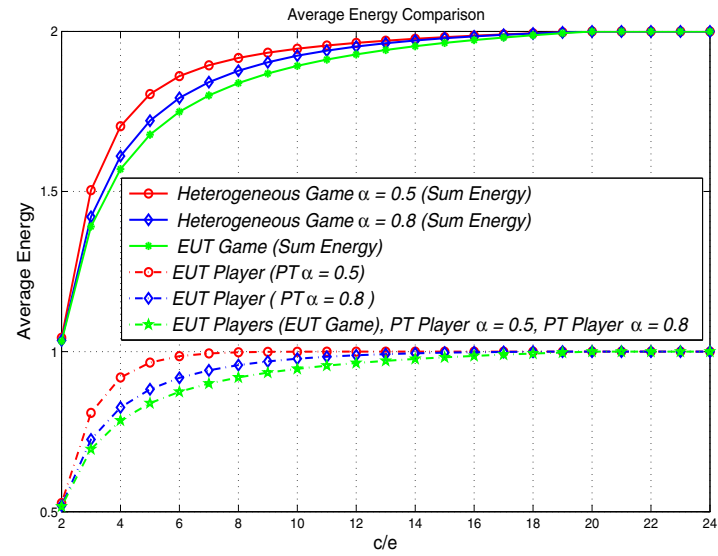

(b) Average energy comparison

Fig. 3. (a) Transmission probability of each player at mixed NE for each game. (b) Average energy comparison at individual player level and system level. Three games (heterogeneous game $(\alpha=0.8)$, heterogeneous game $(\alpha=0.5)$, EUT game) are studied and compared where no delay penalty is considered $(d=0)$. Results in (a) and (b) are shown as a function of $c / e$ where $e=2$ is fixed and $c$ varies.

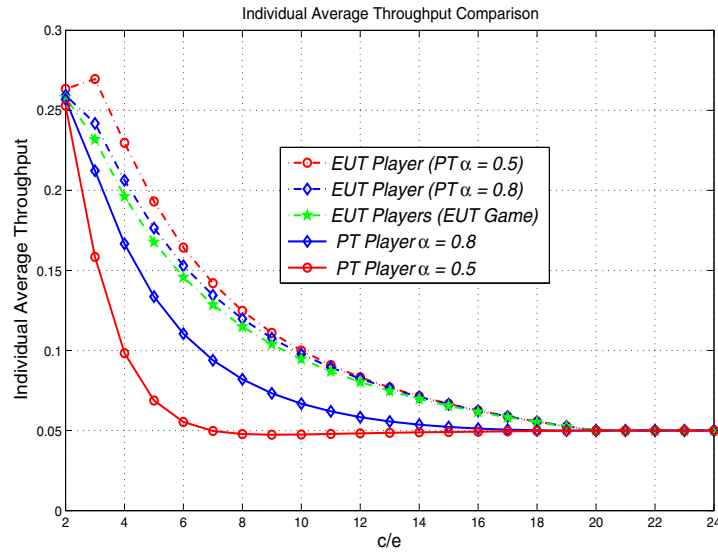

(a) Individual average throughput at mixed $\mathrm{NE}$

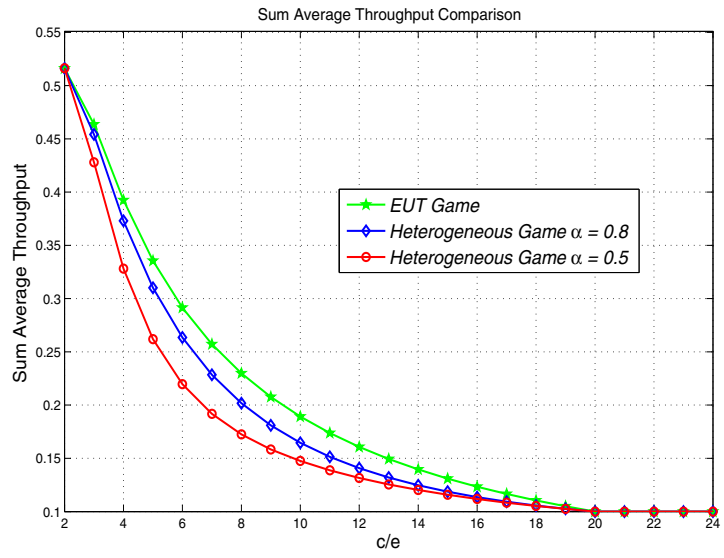

(b) Sum average throughput of both player at mixed NE

Fig. 4. Average throughput comparison at individual player level and system level. Three games (heterogeneous game $(\alpha=0.8)$, heterogeneous game $(\alpha=0.5)$, EUT game) are studied and compared where no delay penalty is considered $(d=0)$. Results in $(c)$ and $(d)$ are shown as a function of $c / e$ where $e=2$ is fixed and $c$ varies.

deviates more from EUT, the above trends are also more exaggerated. Note that, each player's achieved average throughput eventually decreases as the unit throughput reward increases since players also transmit more aggressively and thus generate more collisions. The deviation of one player from EUT to PT can also significantly impact the system level performance as in Fig. 4(b). It can be observed that the players in a heterogeneous game achieve less sum average throughput than those in the homogeneous EUT game. As the PT player deviates more from EUT, the heterogeneous game suffers an even larger sum average throughput loss.

In Fig. 5, we study a heterogeneous game in a more general setting where both unit throughput reward and unit delay penalty are non-zero and varying. For comparison purpose, we choose $c_{1}=c_{2}=c, e_{1}=e_{2}=e=2, d_{1}=d_{2}=d$ and $p_{i \mid\{i\}}=p_{j \mid\{j\}}=0.98, p_{i \mid\{i, j\}}=p_{j \mid\{i, j\}}=0.05$ for both players in both the heterogeneous game and the homogeneous EUT game. The PT player's probability weighting index is $\alpha=0.6$. In Fig. 5(a), it can be seen that the introduction of the PT player causes the EUT player of the heterogeneous game transmit more aggressively than the EUT players in the homogeneous EUT game. Within the heterogeneous game, the EUT player is more aggressive than the PT player. Both players' transmission probabilities converge to 1 when $v_{i \mid\{t, t\}} \geq-d_{i}, i=1,2$. In Fig. 5(b), the impact of the PT player's deviation on the average delay is shown. The EUT player in the heterogeneous game suffers slightly less average delay than the EUT players in the homogeneous EUT game. However, within the heterogeneous game, the PT player suffers more average delay than the EUT player. Further, the deviation of the PT player also causes the heterogeneous game suffer a larger sum average delay than the homogeneous EUT game. In Fig. 5(c), it has been shown that more sum average energy is consumed in the heterogeneous game than that in the homogeneous EUT game. Fig. 5(d) has shown that the heterogeneous game suffers a sum average throughput degradation 


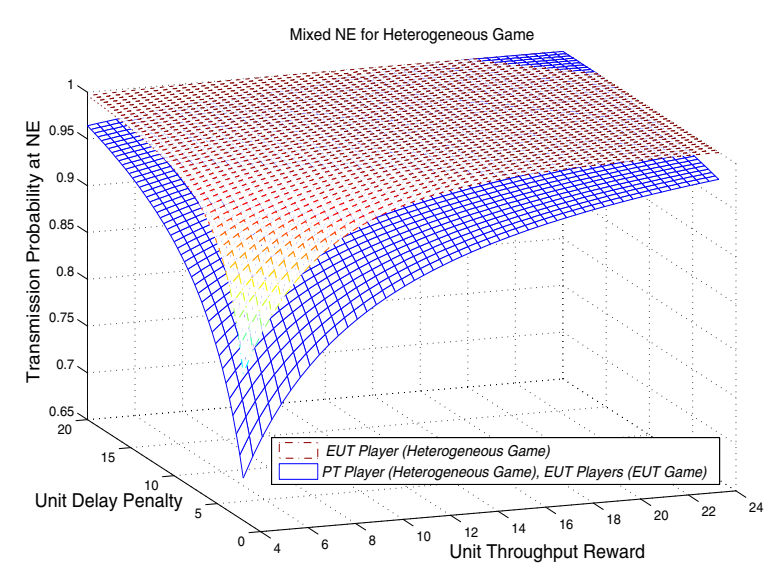

(a) Transmission probability at mixed NE

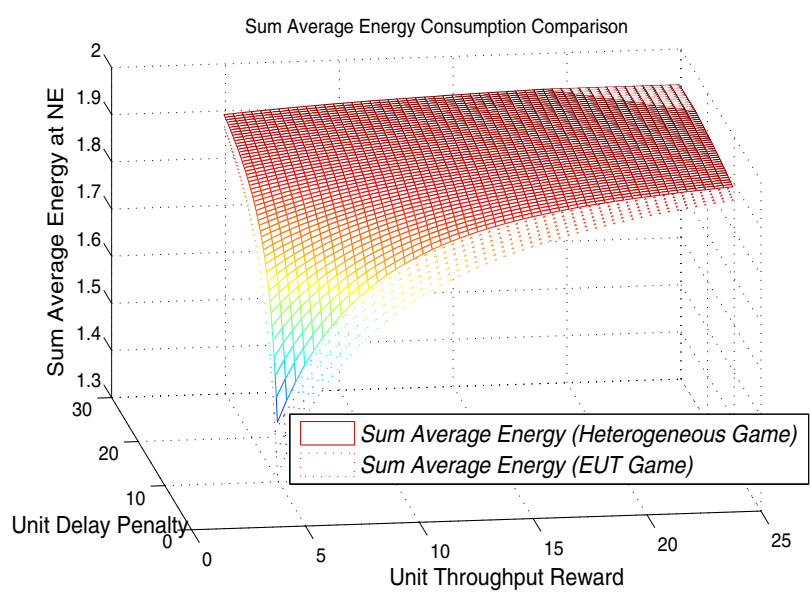

(c) Average Energy at mixed NE

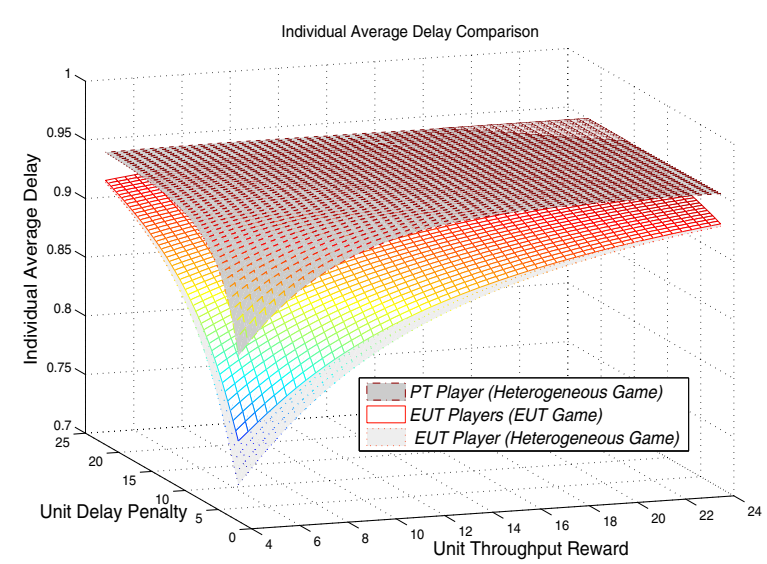

(b) Individual average delay at mixed $\mathrm{NE}$

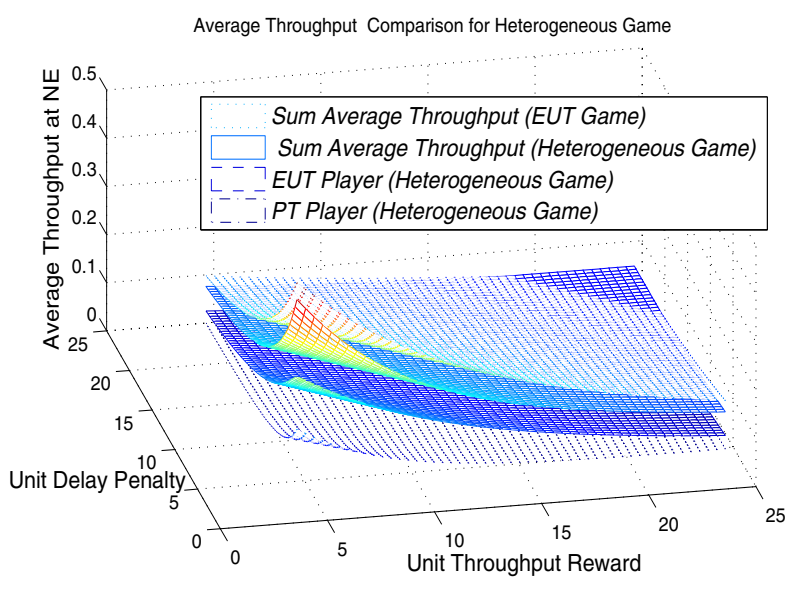

(d) Average Throughput at mixed NE

Fig. 5. A heterogeneous game $(\alpha=0.6)$ and a homogeneous EUT game are compared where unit throughput reward and unit delay penalty are non-zero.

compared to the homogeneous EUT game. Moreover, the PT player is shown to achieve less average throughput than the EUT player within the heterogeneous game.

Therefore, together with the first set of numerical result this subsection has demonstrated 3 main consequences of the PT player's deviation from EUT as listed below:

- (C1) The deviation of PT player causes the EUT player in the heterogeneous game achieve more average throughput, suffer less average delay but consume more average energy than the EUT players in the homogeneous EUT game.

- (C2) Within the heterogeneous game, the PT player achieves less average throughput but suffers more average delay. The EUT player consumes more average energy.

- (C3) The deviation of the PT player undermines the overall system level performance in every aspect as the heterogeneous game achieves less sum average throughput, suffers more sum average delay and consumes more sum average energy than the homogeneous EUT game.

\section{Analytical Insights into Consequence C1 - C3}

We will now prove the three main consequences (1), (2) and (3) in subsection $B$ under mild conditions. We begin with the following Lemmas.
Lemma 3. Each player's average throughput function (10) is an increasing function of her own transmission probability and a decreasing function of her opponent's transmission probability.

Proof: It can be easily verified that $\frac{\partial T_{i}(\mathbf{p})}{\partial p_{i}}=p_{j} p_{i \mid\{i, j\}}+$ $\left(1-p_{j}\right) p_{i \mid\{i\}}>0$ and $\frac{\partial T_{i}(\mathbf{p})}{\partial p_{j}}=p_{i} p_{i \mid\{i, j\}}-p_{i} p_{i \mid\{i\}}<0$ since $p_{i \mid\{i\}}>p_{i \mid\{i, j\}}$ that simply reflects the fact that player $i$ gets a higher successful packet delivery probability for a collision-free transmission than a collision.

Lemma 4. Each player's average delay function (12) is a decreasing function of her own transmission probability and an increasing function of her opponent's transmission probability.

Proof: This also follows the fact that $p_{i \mid\{i\}}>p_{i \mid\{i, j\}}$.

Lemma 5. If $v_{i \mid\{t, t\}}<0, v_{i \mid\{t, n t\}}>0$, there exists a unique number $p^{\text {int }} \in(1 / \rho, 1-1 / \rho)$ ( $\rho$ denotes the Euler's number here) such that $u_{i}^{P T}\left(1, p^{\text {int }}\right)=u_{i}^{E U T}\left(1, p^{\text {int }}\right)$. Further, $u_{i}^{P T}\left(1, p_{j}\right) \leq u_{i}^{E U T}\left(1, p_{j}\right)$ and $u_{i}^{P T}\left(1, p_{j}\right)$ is convex when $p_{j} \in\left[0, p^{\text {int }}\right)$. Meanwhile, $u_{i}^{P T}\left(1, p_{j}\right) \geq u_{i}^{E U T}\left(1, p_{j}\right)$ and $u_{i}^{P T}\left(1, p_{j}\right)$ is concave when $p_{j} \in\left[p^{\text {int }}, 1\right]$.

Proof: Note that, $u_{i}^{P T}(1,1 / \rho)=w_{i}(1 / \rho) v_{i \mid\{t, t\}}+w_{i}(1-$ $1 / \rho) v_{i \mid\{t, n t\}}<1 / \rho \times v_{i \mid\{t, t\}}+(1-1 / \rho) \times v_{i \mid\{t, n t\}}=$ 


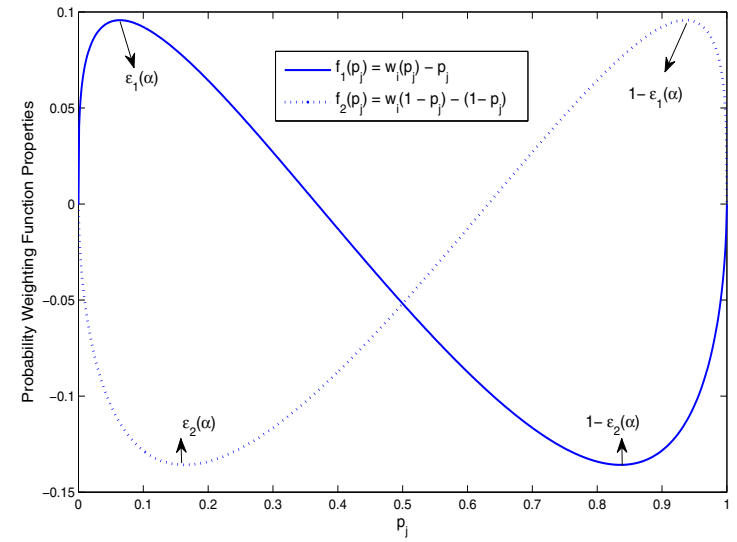

Fig. 6. $w_{i}\left(p_{j}\right)-p_{j}$ and $w_{i}\left(1-p_{j}\right)-\left(1-p_{j}\right)$ as functions of $p_{j}$ for $\alpha=0.6$.

$u_{i}^{E U T}(1,1 / \rho)$. This holds because $v_{i \mid\{t, t\}}<0, v_{i \mid\{t, n t\}}>0$ and the probability weighting function has the property that $w_{i}(1 / \rho)=1 / \rho$ and $w_{i}(1-1 / \rho)<1-1 / \rho$. Similarly, we can show that $u_{i}^{P T}(1,1-1 / \rho)=w_{i}(1-1 / \rho) v_{i \mid\{t, t\}}+$ $w_{i}(1 / \rho) v_{i \mid\{t, n t\}}>(1-1 / \rho) \times v_{i \mid\{t, t\}}+1 / \rho \times v_{i \mid\{t, n t\}}=$ $u_{i}^{E U T}(1,1-1 / \rho)$. Thus, there must exist a point $p^{\text {int }} \in$ $(1 / \rho, 1-1 / \rho)$ such that $u_{i}^{P T}\left(1, p^{\text {int }}\right)=u_{i}^{E U T}\left(1, p^{\text {int }}\right)$ due to the continuities of both $u_{i}^{P T}\left(1, p_{j}\right)$ and $u_{i}^{E U T}\left(1, p_{j}\right)$. We define the difference function of the PT utility and the EUT utility when the player always transmits as $\Delta\left(p_{j}\right)=$ $u_{i}^{P T}\left(1, p_{j}\right)-u_{i}^{E U T}\left(1, p_{j}\right)=f_{1}\left(p_{j}\right) v_{i \mid\{t, t\}}+f_{2}\left(p_{j}\right) v_{i \mid\{t, n t\}}$ where $f_{1}\left(p_{j}\right)=w_{i}\left(p_{j}\right)-p_{j}$ and $f_{2}\left(p_{j}\right)=w_{i}\left(1-p_{j}\right)-\left(1-p_{j}\right)$. According to the definition of probability weighting function (3) and from Fig. 1, it can be easily seen that the function $f_{1}\left(p_{j}\right)$ is a monotonically increasing function in $\left[0, \epsilon_{1}(\alpha)\right]$, decreasing function in $\left(\epsilon_{1}(\alpha), 1-\epsilon_{2}(\alpha)\right)$ and increasing function in $\left[1-\epsilon_{2}(\alpha), 1\right]$. Similarly, $f_{2}\left(p_{j}\right)$ can be shown to be a monotonically decreasing function in $\left[0, \epsilon_{2}(\alpha)\right]$, increasing function in $\left(\epsilon_{2}(\alpha), 1-\epsilon_{1}(\alpha)\right)$ and decreasing function in $\left[1-\epsilon_{1}(\alpha), 1\right]$. Note that, $\epsilon_{1}(\alpha), \epsilon_{2}(\alpha) \in(0,1)$ depend on the probability weighting index $\alpha$ and $\epsilon_{1}(\alpha)<\epsilon_{2}(\alpha)$. Also, it can be shown that $\partial w_{i}\left(p_{j}\right) / \partial p_{j}=1$ when $p_{j}=\epsilon_{1}(\alpha), 1-\epsilon_{2}(\alpha)$. The above properties can be shown in Fig. 6 .

Moreover, for an arbitrary $p_{j} \in\left[0, \epsilon_{1}(\alpha)\right), \Delta\left(p_{j}\right)<0$ since $f_{1}\left(p_{j}\right)>0, f_{2}\left(p_{j}\right)<0, v_{i \mid\{t, t\}}<0$ and $v_{i \mid\{t, n t\}}>0$. $\Delta\left(p_{j}\right)$ decreases further as $p_{j}$ approaches $\epsilon_{1}(\alpha)$ and then there must be a point $\zeta_{1} \in\left[\epsilon_{1}(\alpha), \epsilon_{2}(\alpha)\right]$ where $\Delta\left(p_{j}\right)$ begins to increase. When $p_{j} \in\left[\epsilon_{2}(\alpha), 1-\epsilon_{2}(\alpha)\right], \Delta\left(p_{j}\right)$ keeps increasing further since $d f_{1}\left(p_{j}\right) / d p_{j}<0, d f_{2}\left(p_{j}\right) / d p_{j}>0$ and thus $d \Delta\left(p_{j}\right) / d p_{j}>0$. Note that, $\Delta\left(p^{\text {int }}\right)=0$ and this zero is unique due to the above construction. Hence, we can deduct that $u_{i}^{P T}\left(1, p_{j}\right)$ must lie below $u_{i}^{E U T}\left(1, p_{j}\right)$ and be convex when $p_{j} \in\left[0, p^{\text {int }}\right)$ if we draw $u_{i}^{P T}\left(1, p_{j}\right)$ and $u_{i}^{E U T}\left(1, p_{j}\right)$ together. At the other end, for an arbitrary number $p_{j} \in\left[1-\epsilon_{2}(\alpha), 1\right]$, we can show that $\Delta\left(p_{j}\right)>0$. By following a similar approach as in the previous scenario, we can show that $u_{i}^{P T}\left(1, p_{j}\right)>u_{i}^{E U T}\left(1, p_{j}\right)$ and $u_{i}^{P T}\left(1, p_{j}\right)$ is concave when $p_{i} \in\left[p^{\text {int }}, 1\right]$. The above characteristics can be seen in Fig. 7.

Theorem 6. (Consequence (C1)) In a 2-player heterogeneous game, at the mixed NE, the EUT player achieves more average throughput, suffers less average delay and uses increased average energy, compared to that in a 2-player homogeneous EUT game if the PT player's (player $i$ ) value function is such that $v_{i \mid\{t, n t\}}>-\rho d_{i}+(1-\rho) v_{i \mid\{t, t\}}$.

Proof: In the homogeneous EUT game, the EUT player's (player $j$ ) transmission probability $p_{j}^{\text {EUT }}$ at the mixed NE can be solved by setting $u_{i}^{E U T}\left(1, p_{j}\right)=u_{i}^{E U T}\left(0, p_{j}\right)=-d_{i}$. From Lemma 5, when $p_{j} \in\left[p^{\text {int }}, 1\right], u_{i}^{P T}\left(1, p_{j}\right)$ lies above $u_{i}^{E U T}\left(1, p_{j}\right)$ and is concave. If $v_{i \mid\{t, n t\}}>-\rho d_{i}+(1-$ $\rho) v_{i \mid\{t, t\}}$, it can be easily shown that $p_{j}^{\text {EUT }}=\frac{-d_{i}-v_{i \mid\{t, n t\}}}{v_{i \mid\{t, t\}}-v_{i \mid\{t, n t\}}}>$ $1-1 / \rho$. Correspondingly, the horizontal line $u_{i}^{E U T}\left(0, p_{j}\right)=$ $-d_{i}$ must intersect $u_{i}^{P T}\left(1, p_{j}\right)$ in the heterogeneous game at $p_{j}^{\mathrm{H}}$ where $p_{j}^{\mathrm{H}}>p_{j}^{\text {EUT }}$ (A graphical example can be found in Fig. 7). Meanwhile, since the EUT player (player $j$ ) in the heterogeneous game is identical to the EUT player $j$ in the homogeneous EUT game, it can be devised that $p_{i}^{\mathrm{H}}=p_{i}^{\text {EUT }}$. From Lemma 3, the EUT player's (player $j$ ) average throughput function $T_{j}$ in (10) is an increasing function of her own transmission probability and a decreasing function of her opponent's transmission probability. Thus, the EUT player (player $j$ ) in the heterogeneous game achieves a larger average throughput than in the homogeneous EUT game due to her own transmission probability increase while her opponent's stays unchanged. Similarly, the decline in the EUT player's average delay in the heterogeneous can be explained accordingly. However, due to the EUT player's increasing aggressiveness, she consumes more average energy in the heterogeneous game.

Theorem 7. (Consequence (C2)) In a heterogeneous game, if the PT player (player i) and the EUT player (player $j$ ) only differ in the probability weighting index, i.e., $\alpha_{i}<1$ and $\alpha_{j}=1$ and $v_{i \mid\{t, n t\}}>-\rho d_{i}+(1-\rho) v_{i \mid\{t, t\}}$, it can be shown that $p_{i}^{H}<p_{j}^{H}$. In other words, the PT player transmits less aggressively than the EUT player at the mixed NE. Further, the EUT player achieves more average throughput and suffers less average delay but consumes more average energy than the PT player.

Proof: From Lemma 5, $u_{i}^{P T}(1, p)$ lies above $u_{j}^{E U T}(1, p)$ and is concave when $p \in\left[p^{\text {int }}, 1\right]$. Thus, by following a similar approach in Theorem 6, it can be shown that the PT player transmits less aggressively than the EUT player. Correspondingly, the EUT player incurs more average energy than the PT player in the heterogeneous game. Together with Lemma 3 and 4, the claims regarding the players' average throughput and delay are also established.

Theorem 8. (Consequence (C3)) In a heterogeneous game, at the mixed $N E$, if $p_{i}^{H}>\frac{p_{j \mid\{j\}}}{\sum_{i=1,2}\left(p_{i \mid\{i\}}-p_{i \mid\{i, j\}}\right)}$ for the PT player, the two players achieve less sum average throughput, suffer more sum average delay and consume more sum average energy than those of the corresponding homogeneous EUT game. In other words, the deviation of the PT player harms the system performance in every aspect.

Proof: From Theorem 6, it is already known that in the heterogeneous game, the PT player chooses an identical transmission probability as the EUT players in the homogeneous 


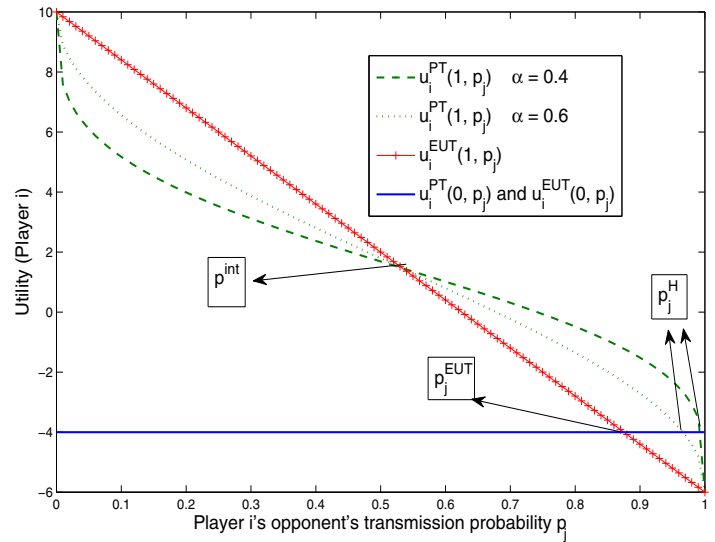

Fig. 7. Players'transmission probabilities at the mixed NE. $v_{i \mid\{t, t\}}=-6$, $v_{i \mid\{t, n t\}}=10$ and $d=-4$.

EUT game as $p_{i}^{\mathrm{H}}=p_{i}^{\mathrm{EUT}}$. However, the EUT player in the heterogeneous game becomes more aggressive than that in the homogeneous EUT game as $p_{j}^{\mathrm{H}}>p_{j}^{\text {EUT }}$. The two players' sum average throughput, sum average delay and sum average energy can be written as:

$$
\begin{aligned}
& T_{s}=p_{i} p_{j} p_{i \mid\{i, j\}}+p_{i}\left(1-p_{j}\right) p_{i \mid\{i\}} \\
& \quad+p_{i} p_{j} p_{j \mid\{i, j\}}+\left(1-p_{i}\right) p_{j} p_{j \mid\{j\}} \\
& D_{s}=p_{i} p_{j}\left(1-p_{i \mid\{i, j\}}\right)+p_{i}\left(1-p_{j}\right)\left(1-p_{i \mid\{i\}}\right)+1-p_{i} \\
& +p_{i} p_{j}\left(1-p_{j \mid\{i, j\}}\right)+\left(1-p_{i}\right) p_{j}\left(1-p_{j \mid\{j\}}\right)+1-p_{j} \\
& E_{s}=p_{i}+p_{j} .
\end{aligned}
$$

Then, it can be easily verified that if $p_{i}>$ $\frac{p_{j \mid\{j\}}}{\sum_{i=1,2}\left(p_{i \mid\{i\}}-p_{i \mid\{i, j\}}\right)}, \partial T_{s} / \partial p_{j}<0$ and $\partial D_{s} / \partial p_{j}>0$. Therefore, the sum average throughput function and sum average delay have been shown as a decreasing and an increasing function of $p_{j}$, respectively. Therefore, if $p_{i}^{\mathrm{H}}>\frac{p_{j \mid\{j\}}}{\sum_{i=1,2}\left(p_{i \mid\{i\}}-p_{i \mid\{i, j\}}\right)}$, then the two players in the heterogeneous game achieve less sum average throughput, suffers more sum average delay and consume more sum energy than that of the homogeneous EUT game due to the increased aggressiveness of the EUT player.

\section{A 2-Player Homogeneous PT Wireless RANDOM ACCESS GAME}

In the above section IV, we studied the scenario when one player deviates from EUT in a 2-player wireless random access game, i.e., a 2-player heterogeneous game. In this section, a more general scenario where both players follow the precept of PT is further analyzed. As in our earlier work [16], a 2-player homogeneous PT wireless random access game has been studied under a $0 / 1$ collision channel model. We now extend this work by considering the homogeneous game under a general collision channel model. Further, without loss of generality, we assume that the two players, i.e., player 1,2, both follow the precepts of PT with probability weighting indices $1>\alpha_{2}>\alpha_{1}>0$. Thus, both players' utility functions should be described as in (9) and the two utility functions differ only in the probability weighting index. Similar to section IV, we will compare the 2-player homogeneous PT game to a benchmark, i.e., the corresponding 2-player homogeneous EUT game. Note that, for player $i=1,2$, her value function regarding a pure strategy profile stays the same with all value functions being linear and about reference point 0 in both the homogeneous PT and EUT games, i.e., $v_{i \mid\{t, t\}}^{P T}=v_{i \mid\{t, t\}}^{E U T}=$ $v_{i \mid\{t, t\}}$ and $v_{i \mid\{t, n t\}}^{P T}=v_{i \mid\{t, n t\}}^{E U T}=v_{i \mid\{t, n t\}}$.

\section{A. The Existence and Uniqueness of Mixed NEs of a Homo- geneous PT Game}

Before investigating the performance deviation of a 2player homogeneous PT game from the corresponding 2player homogeneous EUT game, we first address the existence and uniqueness of the NE.

Theorem 9. There exists a unique mixed $N E\left[p_{1}^{P}, p_{2}^{P}\right]$ for a homogeneous PT game given $v_{i \mid\{t, t\}}<-d_{i}$ and $v_{i \mid\{t, n t\}}>0$ for $i=1,2$.

Proof: The existence of the NE in a homogeneous PT game can be proven by following the same argument in theorem 1. Furthermore, given the identical condition as before, i.e., $v_{i \mid\{t, t\}}<-d_{i}$ and $v_{i \mid\{t, n t\}}>0$ for $i=1,2$, it can be shown that $u_{i}\left(1, p_{j}\right)$ is a decreasing function of $p_{j}$ for $i=1,2$ as shown in (13). Since player $j$ 's mixed strategy at the NE is solved by equating $u_{i}\left(1, p_{j}\right)=u_{i}\left(0, p_{j}\right)=-d_{i}$ and $u_{i}\left(1, p_{j}\right)$ is a strictly decreasing function, $p_{j}^{H}$ must be uniquely determined. Thus, the mixed NE $\left[p_{1}^{\mathrm{P}}, p_{2}^{\mathrm{P}}\right]$ is also unique.

\section{B. A Homogeneous PT Game: More Consequences of Devi- ation from EUT}

In the following, we will compare the performance of the 2-player homogeneous game to that of the 2-player homogeneous EUT game. Specifically, we will study the following issues: (1) Performance differences between two PT players within the 2-player homogeneous PT game. (2) The difference on the performance of the whole system between two games. Without loss of generality, in the 2-player homogeneous PT game, we assume $\alpha_{1}=0.5$ and $\alpha_{2}=0.8$. For both games, two players randomly access the channel with $p_{i \mid\{i\}}=p_{j \mid\{j\}}=$ $0.98, p_{i \mid\{i, j\}}=p_{j \mid\{i, j\}}=0.05, i=1,2$. We begin with a special case where there is no delay penalty for each player as $c_{1}=c_{2}=c, e_{1}=e_{2}=e=2, d_{1}=d_{2}=d=0$. The system performances are studied and compared as a function of $c / e$.

In Fig. 8(a), the PT players in the homogeneous PT game are shown to transmit more aggressively than the EUT players in the homogeneous EUT game. Note that, both the EUT players have the same performance in the homogeneous EUT game due to the same parameter setting. Further, it can be observed that the PT player that deviates less from the EUT (i.e., with choice of $\alpha=0.8$ ) transmits more aggressively in the homogeneous PT game. As a result of the transmission aggressiveness of the PT players at the NE shown in Fig. 8(a), the PT players consume more average energy than the EUT players as shown in Fig. 8(b). Between the PT player, the one with a larger probability weighting index $(\alpha=0.8)$ consumes more average energy than the other PT player. More importantly, the deviation of the PT players from EUT costs 


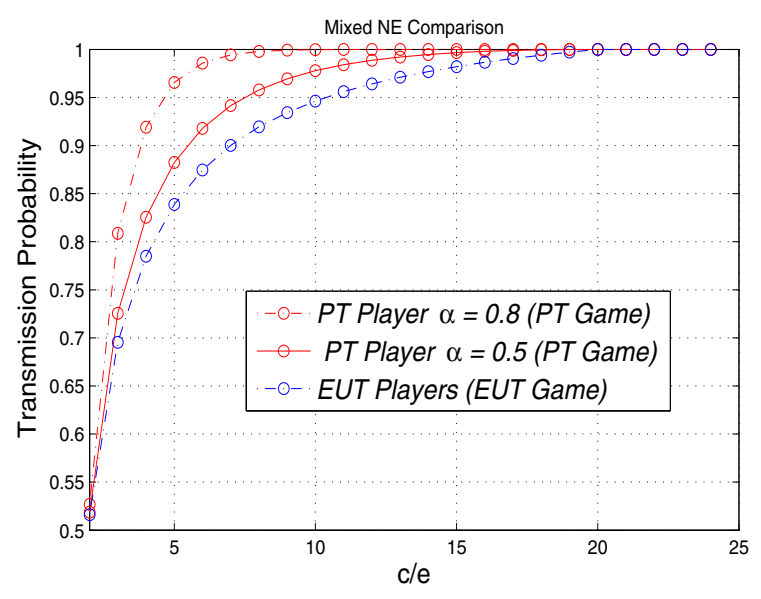

(a) Transmission probability of each player at mixed NE

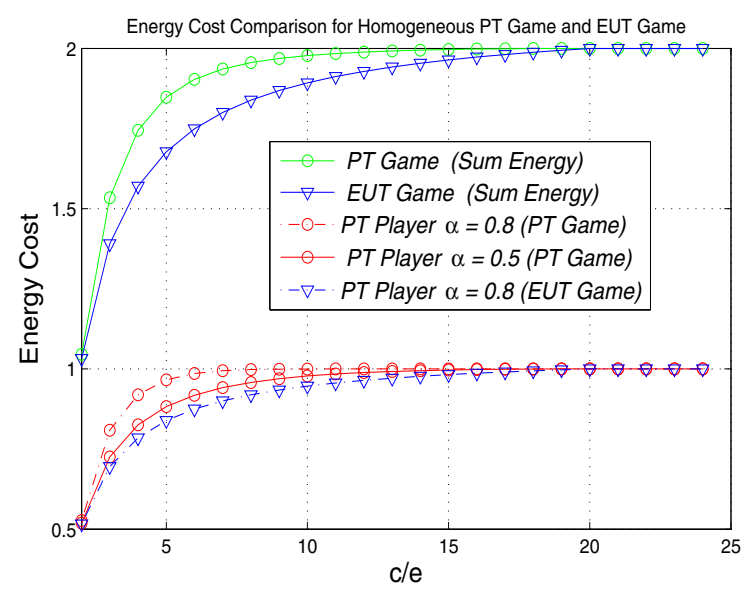

(b) Average energy comparison

Fig. 8. (a) Transmission probability of each player at mixed NE for each game. (b) Average energy comparison at individual player level and system level. Homogeneous PT game $\left(\alpha_{1}=0.5, \alpha_{2}=0.8\right)$ and homogeneous EUT games are studied and compared where no delay penalty is considered $(d=0)$.

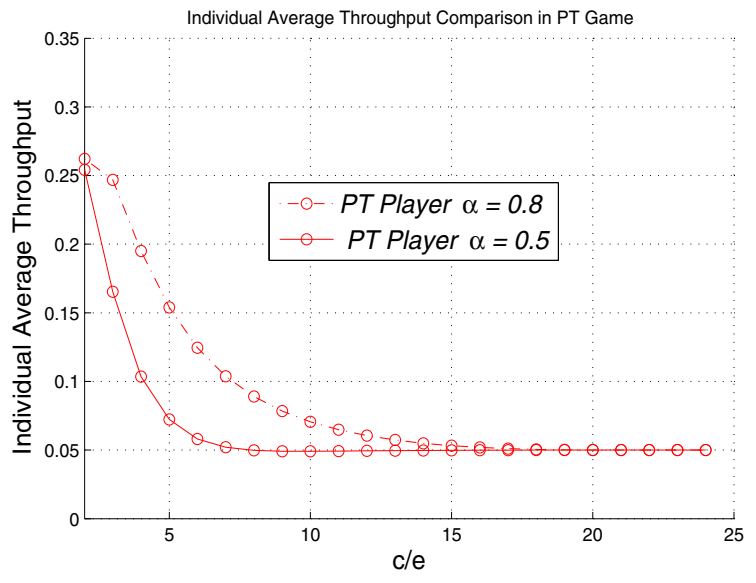

(a) Individual average throughput at mixed NE for homogeneous PT game

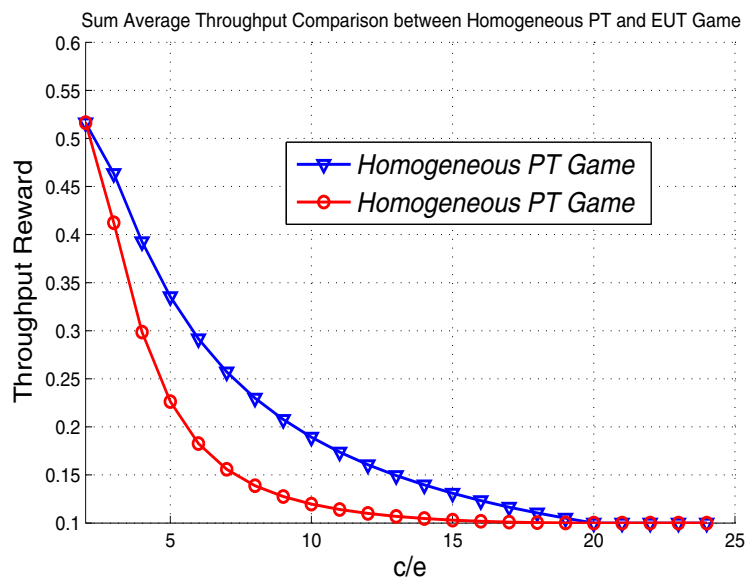

(b) Sum average throughput of both player at mixed NE

Fig. 9. Average throughput comparison at individual player level and system level. Homogeneous PT game $\left(\alpha_{1}=0.5, \alpha_{2}=0.8\right)$ and homogeneous EUT games are studied and compared where no delay penalty is considered $(d=0)$.

the system more energy at the mixed NE compared to the homogeneous EUT game.

In Fig.9(a), the throughput performance difference between two PT players in the homogeneous game is shown. The PT player that deviates less from EUT $(\alpha=0.8)$ achieves more average throughput than the one with a smaller probability weighting index. On the system level, PT players in the homogeneous PT game also incur sum throughput degradation compared to the homogeneous EUT game as shown in Fig.9(b).

While the above results are for the case of no delay penalty, we now study the performance deviation of the PT game in a more general setting. Specifically, we consider a case where unit throughput reward and unit delay penalty both vary while the unit energy cost is fixed, i.e., $c_{1}=c_{2}=c, e_{1}=e_{2}=$ $e=2, d_{1}=d_{2}=d=d$. For comparison, we choose $p_{i \mid\{i\}}=p_{j \mid\{j\}}=0.98, p_{i \mid\{i, j\}}=p_{j \mid\{i, j\}}=0.05, i=1,2$ for PT players and EUT players in both games. In the homogeneous game, we choose $\alpha_{1}=0.5$ and $\alpha_{2}=0.8$ so that PT player 1 deviates more from EUT than PT player 2. In Fig. 10(a), a similar trend in to that in Fig. 8(a) can be observed. The PT players in the homogeneous PT game transmit more aggressively than the EUT players in the homogeneous EUT game. Further, the PT player deviating less from EUT (with a larger $\alpha$ ) transmits more aggressively than the other one. The difference in the transmission aggressiveness decreases as the unit throughput reward and unit delay penalty increase. As a result of the transmission aggressiveness of the PT players at the mixed NE, Fig. 10(b) shows that the homogeneous PT game suffers a sum average throughput degradation compared to the homogeneous EUT game on the system level. This degradation becomes less severe as the unit throughput reward and unit delay penalty increase. Within the homogeneous PT game, the PT player that deviates more from EUT (with a smaller $\alpha$ ) achieves less average throughput than the other one. In Fig. 10(c), homogeneous PT game suffers a greater sum average delay than the EUT game. Within the homogeneous PT game, the PT player who deviates more from EUT (with a smaller $\alpha$ ) suffers a larger average delay than her opponent. Fig. 10(d) shows that the deviation of the PT players of the 


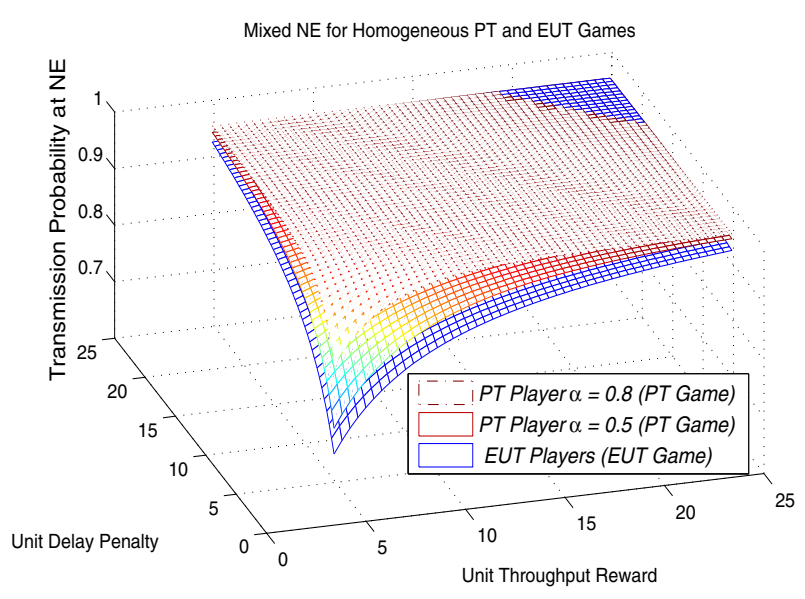

(a) Transmission probability at mixed NE

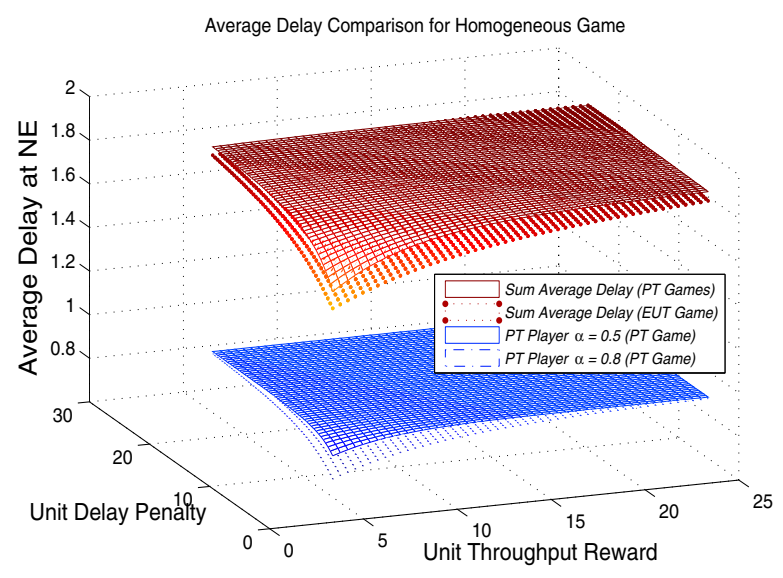

(c) Delay Comparison at mixed NE

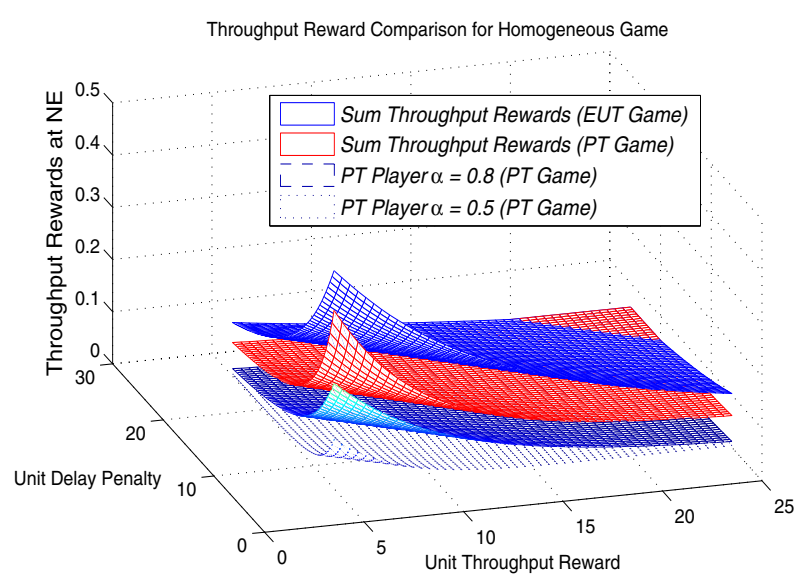

(b) Throughput Comparison at mixed NE

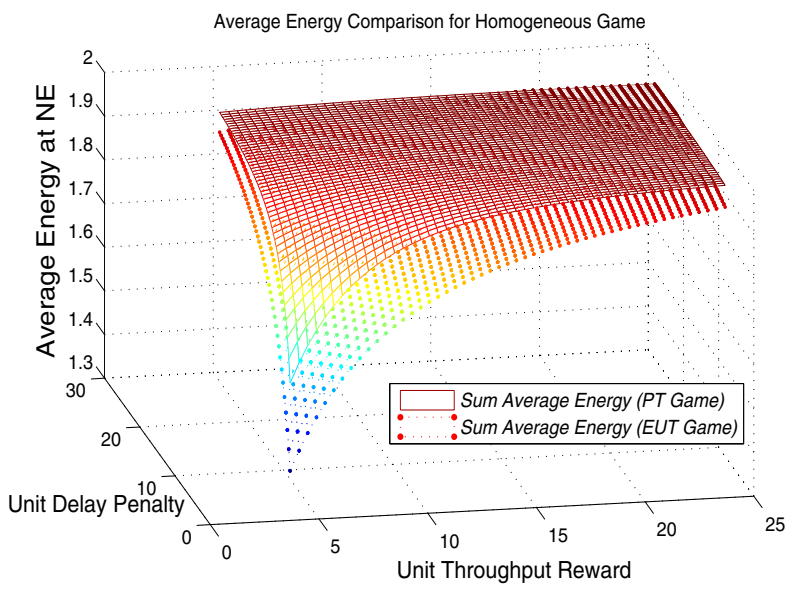

(d) Energy Comparison at mixed NE

Fig. 10. A homogeneous PT game $\left(\alpha_{1}=0.5, \alpha_{2}=0.8\right)$ and a homogeneous EUT game are compared where unit throughput reward and unit delay penalty are non-zero. In each figure, the order of plots are according to the order of the legends from top to bottom.

homogeneous PT game also consumes the system more average energy than that of the EUT game. Since average energy is proportional to a player's transmission aggressiveness, from 10(a), we can see that PT players in homogeneous PT game consumes more average energy than EUT players in the EUT game. Within the homogeneous PT game, the PT player who deviates less from EUT consumes more average energy.

The above results shown in Fig. 8(a)- 10(b) captures the behavior and performance deviation of the PT players in the homogeneous PT game from the homogeneous EUT game. Two distinct features on individual player and system levels can be summarized as below:

- (C4) In the homogeneous PT game, the PT player that deviates less from EUT (with a larger $\alpha$ ) achieves more average throughput and suffers less average delay than the PT player who deviates more from EUT. However, the PT player that deviates less from EUT consumes more average energy.

- (C5) Similar to the observation from the heterogeneous game in section IV, the deviation of both PT players in the homogeneous PT game still hurts the overall system performance in every aspect. The homogeneous PT game achieves less sum average throughput but suffers more average delay and incurs more average energy than the homogeneous EUT game.

\section{Analytical Insights into Consequence C4 - C5}

In the following, we will analyze the above consequences of the deviation of the homogeneous PT game from EUT. The general idea and techniques used here are based on the results in subsection C section IV. The following lemma describes the features of the utility functions of the PT players when they transmit with probability one.

Lemma 10. If $v_{i \mid\{t, t\}}=v_{j \mid\{t, t\}}<0, v_{i \mid\{t, n t\}}=v_{j \mid\{t, n t\}}>$ 0 , there exists a unique number $p^{\text {int }} \in(1 / \rho, 1-1 / \rho)$ ( $\rho$ denotes the Euler's number here) such that $u_{i}^{P T}\left(1, p^{\text {int }}\right)=u_{j}^{P T}\left(1, p^{\text {int }}\right)$. Further, $u_{i}^{P T}\left(1, p_{j}\right) \leq u_{j}^{P T}\left(1, p_{i}\right)$ and $u_{i}^{P T}\left(1, p_{j}\right)$ and $u_{j}^{P T}\left(1, p_{i}\right)$ are convex when $p_{j}, p_{i} \in\left[0, p^{\text {int }}\right)$, respectively. Meanwhile, $u_{i}^{P T}\left(1, p_{j}\right) \geq u_{j}^{P T}\left(1, p_{i}\right)$ and $u_{i}^{P T}\left(1, p_{j}\right)$ and $u_{j}^{P T}\left(1, p_{i}\right)$ are concave when $p_{j}, p_{i} \in\left[p^{\text {int }}, 1\right]$.

Proof: This lemma shows that when both PT players transmit with probability one, the two PT players' utility functions have similar shape, i.e., first convex and then concave. Further, the PT player with a smaller probability weighting 
index $\alpha$ has a utility function of larger curvature than the other PT player that is a direct result from Fig. 1. Note that, $u_{i}^{P T}(1,1 / \rho)=w_{i}(1 / \rho) v_{i \mid\{t, t\}}+w_{i}(1-1 / \rho) v_{i \mid\{t, n t\}}<$ $w_{j}(1 / \rho) \times v_{i \mid\{t, t\}}+w_{j}(1-1 / \rho) \times v_{i \mid\{t, n t\}}=u_{j}^{P T}(1,1 / \rho)$. This holds because $v_{i \mid\{t, t\}}<0, v_{i \mid\{t, n t\}}>0$ and the probability weighting function has the property that $w_{i}(1 / \rho)=1 / \rho$ and $w_{i}(1-1 / \rho)<w_{j}(1-1 / \rho)$. Similarly, we can show that $u_{i}^{P T}(1,1-1 / \rho)=w_{i}(1-1 / \rho) v_{i \mid\{t, t\}}+w_{i}(1 / \rho) v_{i \mid\{t, n t\}}>$ $w_{j}(1-1 / \rho) \times v_{i \mid\{t, t\}}+w_{j}(1 / \rho) \times v_{i \mid\{t, n t\}}=u_{j}^{P T}(1,1-1 / \rho)$. Thus, there must exist a point $p^{\text {int }} \in(1 / \rho, 1-1 / \rho)$ such that $u_{i}^{P T}\left(1, p^{\text {int }}\right)=u_{j}^{P T}\left(1, p^{\text {int }}\right)$ due to the continuities of both $u_{i}^{P T}\left(1, p_{j}\right)$ and $u_{j}^{P T}\left(1, p_{j}\right)$. Similar to the proof of Lemma 5, the rest of the lemma can be proven based on the definition of probability weighting function (3) and from Fig. 1 and the fact that both utility functions above are strictly decreasing functions. The above properties can be seen in Fig. 6.

Theorem 11. (Consequence (C4)) In a 2-player homogeneous $P T$ game, the PT player $i$ who deviates more from EUT (with a smaller probability weighting index $\alpha$ ) achieves less average throughput, suffers more average delay but consumes less average energy than the other PT player $j$. Further, both PT players transmit more aggressively than the EUT players of the corresponding homogeneous EUT game if $v_{i \mid\{t, t\}}=v_{j \mid\{t, t\}}, v_{i \mid\{t, n t\}}=v_{j \mid\{t, n t\}}$ and $v_{i \mid\{t, n t\}}>-\rho d_{i}+$ $(1-\rho) v_{i \mid\{t, t\}}$.

Proof: From Theorem 6, it can be shown that given $v_{i \mid\{t, t\}}=v_{j \mid\{t, t\}}, v_{i \mid\{t, n t\}}=v_{j \mid\{t, n t\}}$ and $v_{i \mid\{t, n t\}}>-\rho d_{i}+$ $(1-\rho) v_{i \mid\{t, t\}}$, at the mixed NE $p_{i}^{\mathrm{P}}, p_{j}^{\mathrm{P}} \in\left[p^{\text {int }}, 1\right]$ and therefore $p_{i}^{\mathrm{P}}<p_{j}^{\mathrm{P}}$ and $p_{i}^{\mathrm{P}}, p_{j}^{\mathrm{P}}>p_{i}^{\text {EUT }}=p_{j}^{\text {EUT }}$. Moreover, from Lemma 3 and 4, it can be concluded that player $i$ achieves less average throughput and suffers more average delay. However, she employs less energy than player $j$ since she transmits less aggressively.

Theorem 12. (Consequence (C5)) Suppose the mixed NE of the homogeneous EUT game $\left[p_{i}^{\text {EUT }}, p_{j}^{\text {EUT }}\right]$ is such that $p_{i}^{\text {EUT }}>$ $\frac{p_{j \mid\{j\}}}{\sum_{i=1,2}\left(p_{i \mid\{i\}}-p_{i \mid\{i, j\}}\right)}$. Then in a 2-player homogeneous PT game, the two PT players achieve less sum average throughput, suffer more sum average delay and consume more sum average energy than those of the corresponding homogeneous EUT game. In other words, the deviation of the PT players in the homogeneous PT game harms the system performance in every aspect.

Proof: From Theorem 8, it is shown that if $p_{j}>$ $\frac{p_{i \mid\{i\}}}{\sum_{i=1,2}\left(p_{i \mid\{i\}}-p_{i \mid\{i, j\}}\right)}, \partial T_{s} / \partial p_{i}<0$ and $\partial D_{s} / \partial p_{i}>0$. Now,

Consider a heterogeneous game derived from the homogeneous PT game where player $j$ becomes an EUT player. Then, $p_{j}^{\mathrm{P}}=p_{j}^{\mathrm{H}}>p_{j}^{\text {EUT }}$ and $p_{i}^{\mathrm{P}}>p_{i}^{\mathrm{H}}$ since player $j$ faces the same opponent in the two games but player $i$ faces different opponents. Therefore, if $p_{j}^{\text {EUT }}>\frac{p_{i \mid\{i\}}}{\sum_{i=1,2}\left(p_{i \mid\{i\}}-p_{i \mid\{i, j\}}\right)}$, it is true that $p_{j}^{\mathrm{P}}>\frac{p_{i \mid\{i\}}}{\sum_{i=1,2}\left(p_{i \mid\{i\}}-p_{i \mid\{i, j\}}\right)}$. Then, from Theorem 8 , sum average throughput and sum average delay will be a decreasing and an increasing function of $p_{i}$ at the mixed NEs, i.e., $\partial T_{s} / \partial p_{i}<0$ and $\partial D_{s} / \partial p_{i}>0$, respectively. Thus, it can be concluded the homogeneous PT game achieves less sum average throughput but suffers more average delay than the heterogeneous game as $p_{i}^{\mathrm{P}}>p_{i}^{\mathrm{H}}$. Together with Theorem
8 , the statement in the theorem can be shown.

\section{N-Player HomogeneOUS GAmes}

We further consider a N-player symmetric homogeneous setting where all the players have identical utility functions and experience the same channel conditions. Note that, the $\mathrm{N}$-player symmetric game reflects a realistic scenario where every player has a collective (aggregated) view of the set of the players in the game. Further, analyzing and evaluating the perceptions of each of the other $N-1$ players is beyond the feasibility of a single end-user's action. We now state the following theorem.

Theorem 13. If each player's utility function (6) is a strictly decreasing function of her opponent's transmission probability and neither $\{t\}$ nor $\{n t\}$ is the pure dominant strategy for each player, there exists a unique mixed NE for an N-player homogeneous wireless random access game with symmetric utility functions.

Proof: The existence of the mixed NEs can be established by generalizing the results in Theorem 1 . Since the game is symmetric, all the players share the identical utility function, i.e., $u_{i}^{P T}(\cdot)=u_{j}^{P T}(\cdot), \forall i \neq j$. Further, each player will choose the identical transmission probability at the mixed NE. Otherwise, assuming there exists a mixed NE such as $\mathbf{p}^{*}=\left[p^{*}, \ldots, p_{i}^{*}, \ldots, p_{j}^{*}, \ldots, p^{*}\right]$ and without loss of generality, two players have different transmission probabilities, i.e., $p_{i}^{*} \neq$ $p_{j}^{*}$, at the mixed NE, we have $u_{i}^{P T}\left(1, \mathbf{p}_{-i}^{*}\right) \neq u_{j}^{P T}\left(1, \mathbf{p}_{-j}^{*}\right)$. However, this contradicts the symmetry condition where at the mixed NE all the players' utilities should be the same, i.e., $-d_{i}=-d, \forall i \in \mathcal{N}$. By following the same rationale, it can be shown that at the mixed NE, each player's transmission probability must be equal. Next, we will show the uniqueness of this symmetric mixed NE. Assuming $\mathbf{p}^{*}=\left[p^{*}, \ldots, p^{*}\right]_{N \times 1}$ and $\mathbf{p}^{\prime}=\left[p^{\prime}, \ldots, p^{\prime}\right]_{N \times 1}$ are two different mixed NEs for the game, we can establish that:

$$
u_{i}^{P T}\left(1, \mathbf{p}_{-i}^{*}\right)=u_{i}^{P T}\left(1, \mathbf{p}_{-i}^{\prime}\right)=-d_{i}, \forall i \in \mathcal{N},
$$

where $\mathbf{p}_{-i}^{*}$ and $\mathbf{p}_{-i}^{\prime}$ denote the $i$-th player's opponent transmission probability vector, i.e., $\mathbf{p}_{-i}^{*}=\left[p^{*}\right]_{(N-1) \times 1}$ and $\mathbf{p}_{-i}^{\prime}=\left[p^{\prime}\right]_{(N-1) \times 1}$. If $p^{*}<p^{\prime}$, it can be shown that $u_{i}^{P T}\left(1, \mathbf{p}_{-i}^{*}\right)>u_{i}^{P T}\left(1, \mathbf{p}_{-i}^{\prime}\right)$ since $u_{i}^{P T}(\cdot)$ is a strictly decreasing function of each $i$-th player's opponent's transmission probability. If $p^{*}>p^{\prime}, u_{i}^{P T}\left(1, \mathbf{p}_{-i}^{*}\right)<u_{i}^{P T}\left(1, \mathbf{p}_{-i}^{\prime}\right)$. Thus, if $p^{*} \neq p^{\prime}$, (19) will not be established and a contradiction exists. Then, the mixed NE of the N-player symmetric homogeneous wireless random access game is shown to be unique. Note that, an N-player symmetric homogeneous EUT game is just a special case where every player's probability weighting index is 1 .

In the following example, we compare the characteristics of mixed NEs of 3-player homogeneous PT and homogeneous EUT games where $\alpha_{i}=0.6, p_{i \mid\{i\}}=1$ and $p_{i \mid \mathcal{J}(\mathbf{a})}=$ $0, \forall \mathcal{J}(\mathbf{a}) \backslash\{i\} \neq \emptyset, \forall i \in \mathcal{N}$. Further, we assume $e=d=2$ and $c$ varies as multiples of $e$. Note that, due to symmetry, the three players in either PT or EUT games have identical performance.

From Fig. 11, it can be observed that the players in the homogeneous EUT game achieve higher individual and thus 


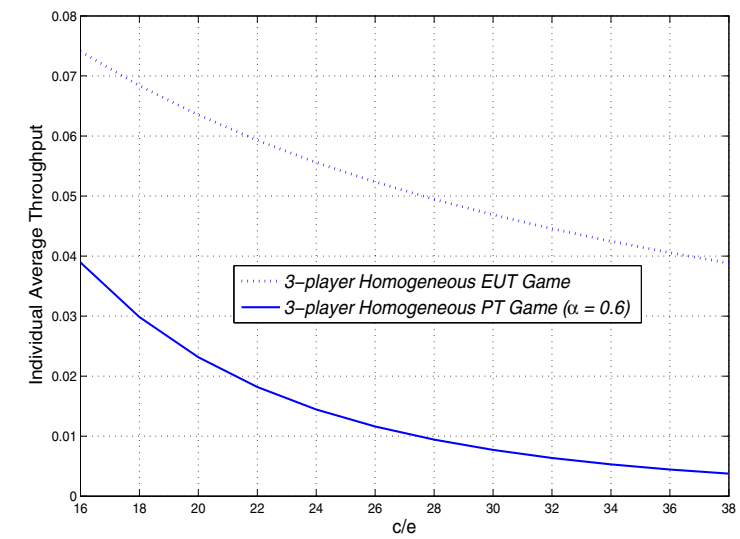

Fig. 11. Average throughput comparison between PT and EUT Games. All the players in the each game have the same average throughput due to the symmetry.

sum average throughput than that in the homogeneous PT game. Moreover, the homogeneous PT game is also observed to have larger individual and sum average delay and average energy consumption than the homogeneous EUT game.

However, the uniqueness of the mixed NE can still be established for the scenario where $N=3$. Assuming $\mathbf{p}_{1}=$ $\left[p_{1}^{*}, p_{2}^{*}, p_{3}^{*}\right]$ and $\mathbf{p}_{2}=\left[p_{1}^{\prime}, p_{2}^{\prime}, p_{3}^{\prime}\right]$ are two different mixed NEs, it can be established that:

$$
\begin{gathered}
u_{1}^{P T}\left(1, p_{2}^{*}, p_{3}^{*}\right)=u_{1}^{P T}\left(1, p_{2}^{\prime}, p_{3}^{\prime}\right)=-d_{1} \\
u_{2}^{P T}\left(p_{1}^{*}, 1, p_{3}^{*}\right)=u_{2}^{P T}\left(p_{1}^{\prime}, 1, p_{3}^{\prime}\right)=-d_{2} \\
u_{3}^{P T}\left(p_{1}^{*}, p_{2}^{*}, 1\right)=u_{3}^{P T}\left(p_{1}^{\prime}, p_{2}^{\prime}, 1\right)=-d_{3} .
\end{gathered}
$$

Without loss of generality, we can assume that $p_{2}^{*}<p_{2}^{\prime}$ and $p_{3}^{*}>p_{3}^{\prime}$. To establish (21), it is necessary that $p_{1}^{*}<p_{1}^{\prime}$. Then, (22) cannot be established since $u_{3}^{P T}\left(p_{1}, p_{2}, 1\right)$ is a strictly decreasing function of $p_{1}$ and $p_{2}$. Thus, (20) - (22) can be simultaneously established only when $\mathbf{p}_{1}=\mathbf{p}_{2}$.

\section{End-User Decision MAKING in a DATA PRICING MODEL}

The previous sections studied random access as an exemplary/illustrative model for PT. However, PT has a historic role in monetary transactions and in keeping with this, we will use a simple data pricing model to illustrate the effect of end-user decision making and its impact on wireless network performance. Specifically, we consider a model as shown in Fig. 12 where a Service Provider (SP) offers prices $\mathbf{r}=\left[r_{1}, r_{2}, \ldots, r_{N}\right]$ for the $N$ end users in the network. The $N$ end users accept the service offers with probability $\mathbf{p}=\left[p_{1}, p_{2}, \ldots, p_{N}\right]$. We consider two pricing schemes, namely Usage Based Pricing (UBP) and Throughput Based Pricing (TBP). In each case, the SP chooses its price vector $\mathbf{r}$ so as to maximizing its revenue $R(\mathbf{p})$.

In the UBP scheme, the SP charges the end users based on their actual usage of the resources. In each time slot, if the $i$-th end user chooses to transmit (accept the service offer), the service provider will charge $r_{i}$ for the data service and on

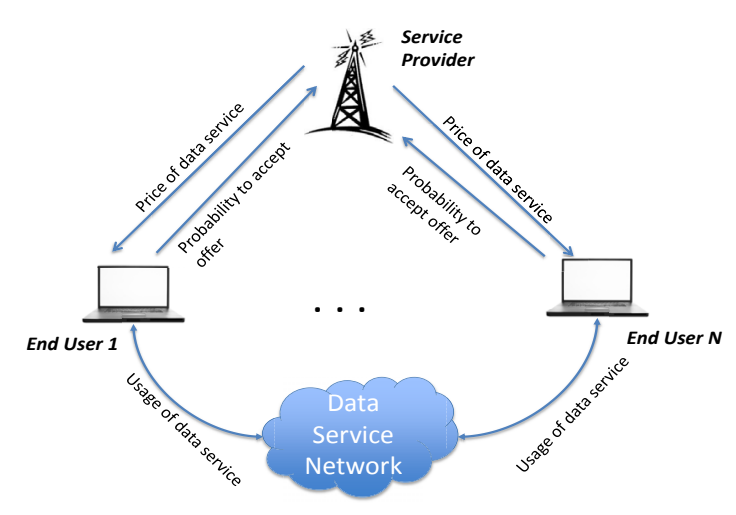

Fig. 12. A two-tier wireless data service pricing model.

average collect a revenue of:

$$
R^{u}(\mathbf{p})=\sum_{i \in \mathcal{N}} r_{i} p_{i}
$$

where $r_{i} p_{i}$ represents the expected fee the service provider can collect from the $i$-th end user.

In the TBP scheme, the SP is allowed to charge each end user in each slot only if her transmission is successful. In each time slot, if the $i$-th end user chooses to use the channel with probability $p_{i}$, then the fee will only be charged proportional to her final throughput. In this case, the exact form of the service provider's revenue is given as:

$$
R^{t}(\mathbf{p})=\sum_{i \in \mathcal{N}} r_{i} p_{i} T_{i},
$$

where $T_{i}=\sum_{\mathbf{a} \in \mathbf{A}}\left(\prod_{j \in \mathcal{J}(\mathbf{a})} p_{j} \prod_{j \in \mathcal{J}^{c}(\mathbf{a})}\left(1-p_{j}\right) p_{i \mid \mathcal{J}(\mathbf{a})}\right)$ is the expected throughput of the $i$-th end user given she chooses to transmit in this time slot.

From the end-user perspective, given the price announced by the service provider, the end users decide to access the data services via a wireless random access channel which can be modeled as a game as shown in section IV. Their final decisions on how to use the services, i.e., the transmission probabilities, are reflected by the mixed NE of the corresponding game. The resulting mixed NE, i.e., the transmission probability vector $\mathbf{p}$, and the price vector $\mathbf{r}$ determine the operating point of the network. Therefore, the mathematical model describing the end-user decision making is similar to the wireless random access game described in the previous sections. However, the end users' value functions for a given strategy vector as shown in Eq. (4) should be modified to reflect the impact of pricing schemes on end users' utilities. Note that, the end users in the data pricing model are also players in the underlying wireless random access game. For the usage based pricing scheme, whenever the $i$-th end user chooses to transmit in the time slot, she will be charged by the service provider $r_{i}$ regardless of the success of the transmission. Therefore, her value function given a strategy 
vector should be modified as follows:

$$
\begin{aligned}
& v_{i \mid \mathbf{a}}^{u}= \\
& \begin{cases}p_{i \mid \mathcal{J}(\mathbf{a})}\left(c_{i}-e_{i}-r_{i}\right)+\left(1-p_{i \mid \mathcal{J}(\mathbf{a})}\right)\left(-e_{i}-d_{i}-r_{i}\right) \\
\quad \text { if } a_{i}=t \\
-d_{i} \quad \text { if } a_{i}=n t,\end{cases}
\end{aligned}
$$

where the notations carry the same physical meaning as in Eq. (4). For the TBP scheme, the end user is charged only for her throughput. No fee will be paid by the end user if the delivery of her packet is not successful. Therefore, the $i$-th end user's value function is:

$$
\begin{aligned}
& v_{i \mid \mathbf{a}}^{t}= \\
& \begin{cases}p_{i \mid \mathcal{J}(\mathbf{a})} & \left(c_{i}-e_{i}-r_{i}\right)+\left(1-p_{i \mid \mathcal{J}(\mathbf{a})}\right)\left(-e_{i}-d_{i}\right) \\
& \text { if } a_{i}=t \\
-d_{i} & \text { if } a_{i}=n t .\end{cases}
\end{aligned}
$$

Note that in this setting, both PT and EUT players retain the same value function as before. In other words, players in a usage based pricing scheme have the same value function as shown in Eq. (25) regardless of their preferences over prospects, either PT or EUT. The same holds true for the throughput based pricing scheme.

To study the impact of end-user decision-making, we consider a 2-user scenario where a SP uses either a UBP or TBP pricing scheme. The SP's utility function in each case is given as:

$$
\begin{aligned}
& u_{s p}^{u}(\mathbf{p})=\sum_{i=1,2} r_{i} p_{i} \\
& u_{s p}^{t}(\mathbf{p})=\sum_{i=1,2} r_{i} p_{i}\left(p_{j} p_{i \mid\{i, j\}}+\left(1-p_{j}\right) p_{i \mid\{i\}}\right),
\end{aligned}
$$

where the $j$-th user refers to the $i$-th user's opponent.

Under the UBP scheme, the end user's value function when she transmits is given as:

$$
\begin{aligned}
v_{i \mid\{t, n t\}}^{u} & =p_{i \mid\{i\}}\left(c_{i}-e_{i}-r_{i}\right)+\left(1-p_{i \mid\{i\}}\right)\left(-e_{i}-d_{i}-r_{i}\right) \\
v_{i \mid\{t, t\}}^{u} & =p_{i \mid\{i, j\}}\left(c_{i}-e_{i}-r_{i}\right)+\left(1-p_{i \mid\{i, j\}}\right)\left(-e_{i}-d_{i}-r_{i}\right) .
\end{aligned}
$$

Similarly, the value functions for the TBP scheme are as follows:

$$
\begin{aligned}
v_{i \mid\{t, n t\}}^{t} & =p_{i \mid\{i\}}\left(c_{i}-e_{i}-r_{i}\right)+\left(1-p_{i \mid\{i\}}\right)\left(-e_{i}-d_{i}\right) \\
v_{i \mid\{t, t\}}^{t} & =p_{i \mid\{i, j\}}\left(c_{i}-e_{i}-r_{i}\right)+\left(1-p_{i \mid\{i, j\}}\right)\left(-e_{i}-d_{i}\right) .
\end{aligned}
$$

Further, the end user's utility functions are given as follows:

$$
\begin{array}{r}
u_{i, u}^{P T}(\mathbf{p})=p_{i} w_{i}\left(p_{j}\right) v_{i \mid\{t, t\}}^{u}+p_{i} w_{i}\left(1-p_{j}\right) v_{i \mid\{t, n t\}}^{u}(33) \\
+\left(1-p_{i}\right)\left(-d_{i}\right) \\
u_{i, t}^{P T}(\mathbf{p})=p_{i} w_{i}\left(p_{j}\right) v_{i \mid\{t, t\}}^{t}+p_{i} w_{i}\left(1-p_{j}\right) v_{i \mid\{t, n t\}}^{t}(34) \\
+\left(1-p_{i}\right)\left(-d_{i}\right) \\
+\left(1-p_{i}\right)\left(-d_{i}\right) \\
(35) \\
u_{i, u}^{E U T}(\mathbf{p})=p_{i} p_{j} v_{i \mid\{t, t\}}^{u}+p_{i}\left(1-p_{j}\right) v_{i \mid\{t, n t\}}^{u}+\left(1-p_{i}\right)\left(-d_{i}\right) \\
u_{i, t}^{E U T}(\mathbf{p})=p_{i} p_{j} v_{i \mid\{t, t\}}^{t}+p_{i}\left(1-p_{j}\right) v_{i \mid\{t, n t\}}^{t}+(1-36)
\end{array}
$$

where $u_{i, u}^{P T}(\mathbf{p})$ and $u_{i, t}^{P T}(\mathbf{p})$ denote a PT user's utility function under UBP and TBP schemes, respectively. Correspondingly, $u_{i, u}^{E U T}(\mathbf{p})$ and $u_{i, t}^{E U T}(\mathbf{p})$ refer to an EUT player's utility functions under both the data pricing schemes, respectively. Mathematically, an EUT user's utility function can be considered as a special case of a PT user's, i.e., the probability weighting index $\alpha=1$. For each data pricing scheme, we will consider 2 scenarios: (1) both end users are EUT users (denoted as UBP-EUT or TBP-EUT) and (2) both end users are PT users (denoted as UBP-PT or TBP-PT). In UBP the SP maximizes Eq. (27) and end users maximize Eq. (35) or Eq. (33). In TBP the SP maximizes Eq. (28) and end users maximize Eq. (36) or Eq. (34).

\section{A. Existence and Uniqueness of the Operating Point}

According to the structure of the data pricing model, the existence and uniqueness of the operating point is determined by the relationship among service provider's price and end users' utility functions. Furthermore, given a service provider's price vector $\mathbf{r}$, the existence and uniqueness of the operating point is equivalent to that of the mixed $\mathrm{NE}$ of the end-user wireless random access game. Therefore, the conclusions on the existence and uniqueness of the operating point can be established as follows.

Theorem 14. There exists a unique operating point $\left[\mathbf{r}^{*}, \mathbf{p}^{*}\right]$ for the UBP with either PT end users or EUT end users if $v_{i \mid\{t, n t\}}^{u}>0$ and $v_{i \mid\{t, t\}}^{u}<-d_{i}$ for $i=1,2$.

Proof: The scenarios where both users are EUT, i.e., UBP-EUT, can be mathematically considered as special cases of the scenario where both end users are PT users. Thus, we will establish the existence and uniqueness of the operating point for UBP-PT and that of the other scenario follows. From theorem 9, it can be shown that, for a fixed a pricing vector $\mathbf{r}=\left[r_{1}, r_{2}\right]$, if $v_{i \mid\{t, n t\}}^{u}>0$ and $v_{i \mid\{t, t\}}^{u}<-d_{i}$ for $i=1,2$, there exists a unique mixed NE $\left[p_{1}^{*}, p_{2}^{*}\right]$ in the corresponding end user level game, i.e, a homogeneous PT game. By applying Eq. (29) and Eq. (30) in the conditions, we can conclude that if $v_{i \mid\{t, n t\}}^{u}=p_{i \mid\{i\}}\left(c_{i}-e_{i}\right)+\left(1-p_{i \mid\{i\}}\right)\left(-e_{i}-d_{i}\right)>r_{i}$ and $p_{i \mid\{i, j\}}\left(c_{i}-e_{i}\right)+\left(1-p_{i \mid\{i, j\}}\right)\left(-e_{i}-d_{i}\right)>r_{i}-d_{i}$, there exists a unique operating point in UBP-PT. The same argument holds true for UBP-EUT.

Theorem 15. There exists a unique operating point $\left[\mathbf{r}^{*}, \mathbf{p}^{*}\right]$ for the TBP with either PT end users or EUT end users if $v_{i \mid\{t, n t\}}^{t}>0$ and $v_{i \mid\{t, t\}}^{t}<-d_{i}$ for $i=1,2$.

The proof of the above theorem follows using similar steps as in the proof of theorem 14 .

\section{B. Impact and Influence of End-User Behavior on UBP}

We begin by providing some analytical results on the UBP scheme.

Lemma 16. An end user's utility when she chooses to transmit, i.e., $u_{i, u}^{P T}\left(1, p_{j}\right)$ or $u_{i, u}^{E U T}\left(1, p_{j}\right)$, is a decreasing function of the charged price $r_{i}$ given a fixed $p_{j}$.

Proof: Under the usage based pricing scheme, whenever the end user chooses to transmit, she will be charged a 
unit price $r_{i}$. A larger value of $r_{i}$ will result a less value of the user's utility. Moreover, given a fixed $p_{j}$, it is true that $\partial u_{i, u}^{P T}\left(1, p_{j}\right) / \partial r_{i}=-\left(w_{i}\left(p_{j}\right)+w_{i}\left(1-p_{j}\right)\right)<0$. In particular, $\partial u_{i, u}^{E U T}\left(1, p_{j}\right) / \partial r_{i}=-1<0$. Thus, the statement is established.

The above lemma 16 can provide us with some hint about the influence of the price $\mathbf{r}$ to the transmission probabilities. The transmission probabilities at the mixed NE are determined by solving $u_{i, u}^{P T}\left(1, p_{j}\right)=-d_{i}$ or $u_{i, u}^{E U T}\left(1, p_{j}\right)=-d_{i}$. Thus, a decrease in the value of $u_{i, u}^{P T}\left(1, p_{j}\right)$ or $u_{i, u}^{E U T}\left(1, p_{j}\right)$ must result in smaller transmission probabilities at the mixed NE.

Theorem 17. End users' transmission probabilities $p_{1}, p_{2}$ will be a decreasing function of the price $r$, i.e., end users will choose a lower service utilization level if service provider charges a larger price for both UBP-PT and UBP-EUT if $v_{i \mid\{t, n t\}}^{u}>0$ and $v_{i \mid\{t, t\}}^{u}<-d_{i}$ for $i=1,2$.

Proof: End user $i$ 's transmission probability $p_{i}$ at the operation point can be determined by solving $\mathcal{F}\left(r, p_{i}(r)\right)=$ $v_{j \mid\{t, n t\}}^{u} w_{j}\left(1-p_{i}\right)+v_{j \mid\{t, t\}}^{u} w_{j}\left(p_{i}\right)+d_{j}=0$. By differentiating both sides with regard to $r$, it can be shown that $\partial \mathcal{F} / \partial r+\partial \mathcal{F} / \partial p_{i} \cdot d p_{i} / d r=0$. Finally, it is true that

$$
\begin{aligned}
d p_{i} / d r & =-(\partial \mathcal{F} / \partial r) /\left(\partial \mathcal{F} / \partial p_{i}\right) \\
& =\frac{w_{j}\left(p_{i}\right)+w_{j}\left(1-p_{i}\right)}{v_{j \mid\{t, n t\}}^{u} \partial w_{j}\left(1-p_{i}\right) / \partial p_{i}+v_{j \mid\{t, t\}}^{u} \partial w_{j}\left(p_{i}\right) / \partial p_{i}}<0,
\end{aligned}
$$

where $w_{j}\left(p_{i}\right)+w_{j}\left(1-p_{i}\right)>0$ and $v_{j \mid\{t, n t\}}^{u} \partial w_{j}\left(1-p_{i}\right) / \partial p_{i}+$ $v_{j \mid\{t, t\}}^{u} \partial w_{j}\left(p_{i}\right) / \partial p_{i}<0$ as shown in theorem 14.

Lemma 18. $P T$ users in UBP-PT will use the data service in a less aggressive manner than the EUT users in UBP-EUT if $v_{i \mid\{t, n t\}}^{u}>(1+\rho) v_{i \mid\{t, t\}}^{u}+\rho d_{i}, i=1,2$.

Proof: The EUT users' transmission probabilities in UBP-EUT can be easily determined as $p_{i}^{E U T}=p_{j}^{E U T}=$ $p^{E U T}=\frac{-d-v_{i \mid\{t, n t\}}^{u}}{v_{i \mid\{t, t\}}^{u}-v_{i \mid\{t, n t\}}^{u}}$. From lemma 5, it can be shown that $u_{i, u}^{P T}\left(1, p_{j}\right) \stackrel{i\{t, t\}}{\leq} u_{i, u}^{E U T}\left(1, p_{j}\right)$ and $u_{i, u}^{P T}\left(1, p_{j}\right)$ is convex when $p^{E U T}<1 / \rho$. As a result, the horizontal line $u_{i, u}^{P T}\left(0, p_{j}\right)=$ $u_{i, u}^{E U T}\left(0, p_{j}\right)=-d_{i}$ must intersect with $u_{i, u}^{P T}\left(1, p_{j}\right)$ at a point $p_{j}^{P T}<p_{j}^{E U T}=p^{E U T}$. Thus, the PT users will transmit less aggressively than the EUT users in this scenario. Further, $p^{E U T}<1 / \rho$ is equivalent to $v_{i \mid\{t, n t\}}^{u}>(1+\rho) v_{i \mid\{t, t\}}^{u}+\rho d_{i}$.

Corollary 19. If $v_{i \mid\{t, n t\}}^{u}=v_{j \mid\{t, n t\}}^{u}$ and $v_{i \mid\{t, t\}}^{u}=v_{j \mid\{t, t\}}^{u}$, the service provider will obtain larger revenue when facing $P T$ users (UBP-PT) than that when facing EUT users (UBPEUT) given $v_{i \mid\{t, n t\}}^{u}>-\rho d_{i}+(1-\rho) v_{i \mid\{t, t\}}^{u}$. If $v_{i \mid\{t, n t\}}>$ $(1+\rho) v_{i \mid\{t, t\}}+\rho d_{i}$, the trend is reversed and the service provider will obtain more revenue from EUT users in UBPEUT.

Proof: The service provider's revenue is defined as $u_{s p}^{u}(\mathbf{p})=\sum_{i=1,2} r_{i} p_{i}$. Thus, given the same $r$, the revenue is totally determined by the transmission probabilities of end users. Thus, with the results in theorem 11 and lemma 18, the statement can be established.

Theorem 20. The service provider's revenue collected from UBP-EUT is a strictly concave function of price $r$. As a result,

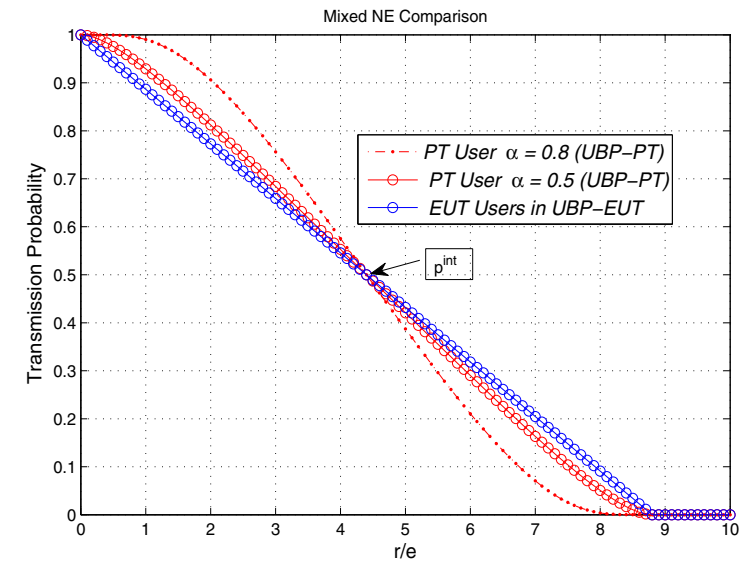

Fig. 13. Transmission probabilities at the operation point for UBP.

there exists a unique optimal operating point in UBP-EUT where the service provider obtains the largest revenue.

Proof: The second order derivative of service provider's revenue from the $i$-th EUT user to the price can be derived as $\partial^{2}\left(r p_{i}\right) / \partial r^{2}=2 \partial p_{i} / \partial r+d^{2} p_{i} / d r^{2}$. Moreover, $p_{i}=\frac{-d-v_{j \mid\{t, n t\}}^{u}}{v_{j \mid\{t, t\}}^{u}-v_{j \mid\{t, n t\}}^{u}}$. Thus, it can be easily shown that $\partial^{2}\left(r p_{i}\right) / \partial r^{2}=2 /\left(v_{j \mid\{t, t\}}^{u}-v_{j \mid\{t, n t\}}^{u}\right)<0$. In other words, the service provider's revenue from the $i$-th user is a strictly concave function of the price $r$ and a unique optimal price can be found by equating $\partial\left(r p_{i}\right) / \partial r=p_{i}+r /\left(v_{j \mid\{t, t\}}^{u}-v_{j \mid\{t, n t\}}^{u}\right)=$ 0 , i.e., $r^{*}=v_{j \mid\{t, n t\}}^{u}+d_{j}$. A similar conclusion can be drawn for the revenue collected from the $j$-th user. Hence, given $v_{i \mid\{t, n t\}}^{u}=v_{j \mid\{t, n t\}}^{u}$ and $v_{i \mid\{t, t\}}^{u}=v_{j \mid\{t, t\}}^{u}$, a unique optimal price can be obtained as $r^{*}=v_{j \mid\{t, n t\}}^{u}+d_{j}=v_{i \mid\{t, n t\}}^{u}+d_{i}$.

While we do not have an analytical proof, we note that our numerical simulations in Fig. 15 do indeed reveal the existence of a unique optimal point for the case of UBP-PT.

We now discuss the analytical insights obtained from lemma 16, theorem 17, lemma 18, corollary 19 and theorem 20 along with some numerical results. Specifically, in UBP-PT, we assume $\alpha_{1}=0.5$ and $\alpha_{2}=0.8$. For both UBP-EUT and UBP-PT, two users randomly access the channel with $p_{i \mid\{i\}}=p_{j \mid\{j\}}=0.98, p_{i \mid\{i, j\}}=p_{j \mid\{i, j\}}=0.1, i=1,2$. We begin with a special case where there is no delay penalty for each user as $c_{1}=c_{2}=c=20, e_{1}=e_{2}=e=2, d_{1}=$ $d_{2}=d=0$ and the price charged varies, i.e., $r_{1}=r_{2}=r$. As a result, $v_{i \mid\{t, n t\}}^{u}=v_{j \mid\{t, n t\}}^{u}$ and $v_{i \mid\{t, t\}}^{u}=v_{j \mid\{t, t\}}^{u}$. The system performances are studied and compared as a function of $r / e$.

The qualitative argument in lemma 16 can be shown as in the following figure. In Fig. 13, it can be seen that, in both UBP-EUT and UBP-PT, end users' transmission probabilities at the operating point decreases as price $r$ increases. Further, as proven in theorem 11, PT users in UBP-PT transmit more aggressively given $v_{i \mid\{t, n t\}}^{u}>-\rho d_{i}+(1-\rho) v_{i \mid\{t, t\}}^{u}, v_{i \mid\{t, n t\}}^{u}=$ $v_{j \mid\{t, n t\}}^{u}$ and $v_{i \mid\{t, t\}}^{u}=v_{j \mid\{t, t\}}^{u}$. This indicates that the service provider will expect a lower service utilization level if she charges a larger price if $v_{i \mid\{t, n t\}}^{u}>0$ and $v_{i \mid\{t, t\}}^{u}<-d_{i}$ for $i=1,2$. This argument holds true for both EUT user and PT 


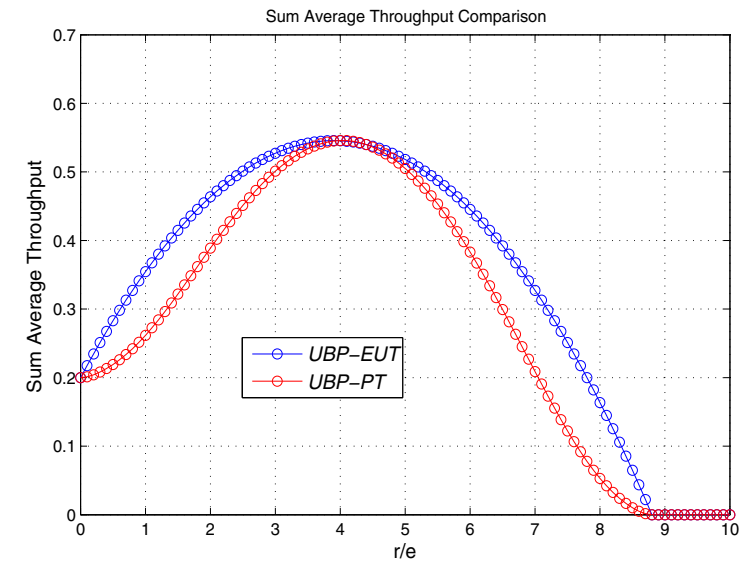

Fig. 14. Sum average throughput at the operation point for UBP.

user scenarios and can be analytically proven in theorem 17 . Another interesting observation in Fig. 13 is that when the price charged becomes relatively large, the PT users in UBPPT will choose a more conservative utilization of the data services. In particular, there exists a transmission probability where $p_{i}^{E U T}=p_{j}^{E U T}=p_{i}^{P T}=p_{j}^{P T}=p^{\text {int }}$ as shown lemma 5 . This phenomenon can be analytically proven in lemma 18 using a similar approach to that used in theorem 11.

In Fig. 14, the sum average throughput of both users are compared between UBP-EUT and UBP-PT. The result shows that with usage based data pricing scheme, the deviation of PT users from EUT still hurts the system performance in terms of throughput. This is actually a direct result from theorem 12. In Fig. 15, service provider's revenues are shown for both UBPEUT and UBP-PT as she charges varying prices to the end users. It can be seen that the service provider will achieve greater revenues when users are PT, even while charging a comparatively lower fee.

The important result from theorem 20 that is shown in Fig. 15 is the difference of optimal operating points between UBPPT and UBP-EUT. It can be seen that both UBP-PT and UBPEUT have unique optimal operating points. Meanwhile, the optimal revenue collected from UBP-PT is slightly larger than that from UBP-EUT. However, the optimal price charged in UBP-PT is slightly smaller than that in UBP-EUT.

In conclusion, for UBP, the type of the underlying users will influence system performance and service provider's revenue. First, a larger price will discourage the users from using the data service for both UBP-EUT and UBP-PT. Second, the deviation of PT users from EUT still degrades the system sum average throughput under the data pricing scheme. Third, under mild conditions as in corollary 19 , the service provider will gain more revenue in UBP-PT than that in UBP-EUT for a small price. However, as the price increases, the trend will be reversed. Finally, the service provider will find a unique optimal price to collect the most revenue in both UBP-PT and UBP-EUT. The optimal revenue collected from UBP-PT is slightly larger than that in UBP-EUT and the the optimal price in UBP-PT is slightly smaller than that in UBP-EUT.

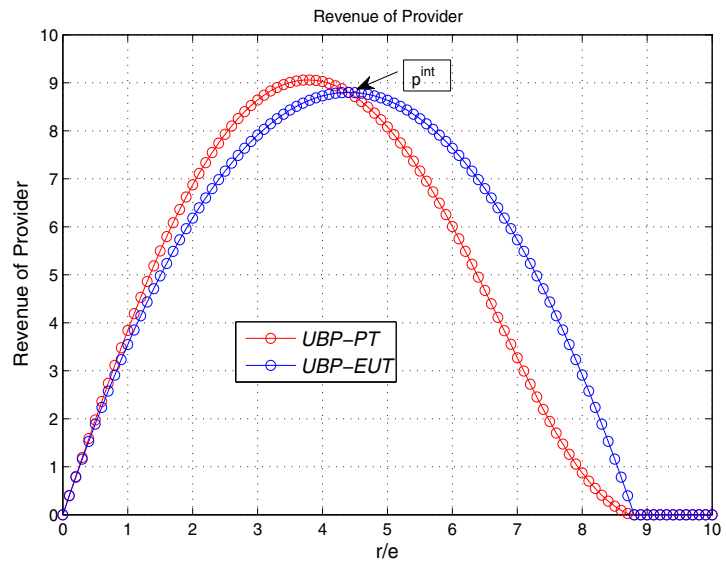

Fig. 15. Service provider's revenue for UBP.

\section{Impact and Influence of End-User Behavior on TBP}

Unlike the UBP scheme, the throughput based pricing scheme only charges the end users for successful transmissions. In the following, we will compare the service provider's revenue and system performance between two scenarios, i.e., the end users are both PT (TBP-PT) and the end users are both EUT (TBP-EUT).

In the UBP scheme, we have shown that a larger price charged by the service provider will discourage the end users from using the data services for both PT and EUT users. The same argument can also be established for the TBP scheme as in the following lemma 21 and theorem 22.

Lemma 21. An end user's utility when she chooses to transmit, i.e., $u_{i, t}^{P T}\left(1, p_{j}\right)$ or $u_{i, t}^{E U T}\left(1, p_{j}\right)$, is a decreasing function of the charged price $r_{i}$ given a fixed $p_{j}$.

Proof: Given a fixed $p_{j}$, it is true that $\partial u_{i, t}^{P T}\left(1, p_{j}\right) / \partial r_{i}=$ $-\left(p_{i \mid\{i, j\}} w_{i}\left(p_{j}\right)+p_{i \mid\{i\}} w_{i}\left(1-p_{j}\right)\right)<0$. In particular, $\partial u_{i, t}^{E U T}\left(1, p_{j}\right) / \partial r_{i}=-p_{i \mid\{i, j\}} p_{j}-p_{i \mid\{i\}}\left(1-p_{j}\right)<0$. Thus, the statement is established.

Theorem 22. End users' transmission probabilities $p_{1}, p_{2}$ will be a decreasing function of the price $r$, i.e., end users will choose a lower service utilization level if service provider charges a larger price for both TBP-PT and TBP-EUT.

Proof: Similar to the proof of theorem 17, it is true that

$$
d p_{i} / d r=\frac{w_{j}\left(p_{i}\right) p_{i \mid\{i, j\}}+w_{j}\left(1-p_{i} p_{i \mid\{i\}}\right)}{v_{j \mid\{t, n t\}}^{t} \partial w_{j}\left(1-p_{i}\right) / \partial p_{i}+v_{j \mid\{t, t\}}^{t} \partial w_{j}\left(p_{i}\right) / \partial p_{i}}<0,
$$

where $w_{j}\left(p_{i}\right) p_{i \mid\{i, j\}}+w_{j}\left(1-p_{i}\right) p_{i \mid\{i\}}>0$ and $v_{j \mid\{t, n t\}}^{t} \partial w_{j}\left(1-p_{i}\right) / \partial p_{i}+v_{j \mid\{t, t\}}^{t} \partial w_{j}\left(p_{i}\right) / \partial p_{i}<0$ as shown in theorem 15.

Theorem 23. If $v_{i \mid\{t, n t\}}^{t}=v_{j \mid\{t, n t\}}^{t}$ and $v_{i \mid\{t, t\}}^{t}=v_{j \mid\{t, t\}}^{t}$, the service provider will obtain a larger revenue when facing EUT users (TBP-EUT) than that when facing PT users (TBP-PT).

Proof: From theorem 11 and 12, it is true that given $v_{i \mid\{t, n t\}}^{t}>-\rho d_{i}+(1-\rho) v_{i \mid\{t, t\}}^{t}, v_{i \mid\{t, n t\}}^{t}=v_{j \mid\{t, n t\}}^{t}$ and $v_{i \mid\{t, t\}}^{t}=v_{j \mid\{t, t\}}^{t}$, TBP-EUT will obtain a larger sum average throughput than that in TBP-PT. Moreover, $u_{s p}^{t}(\mathbf{p})=$ 


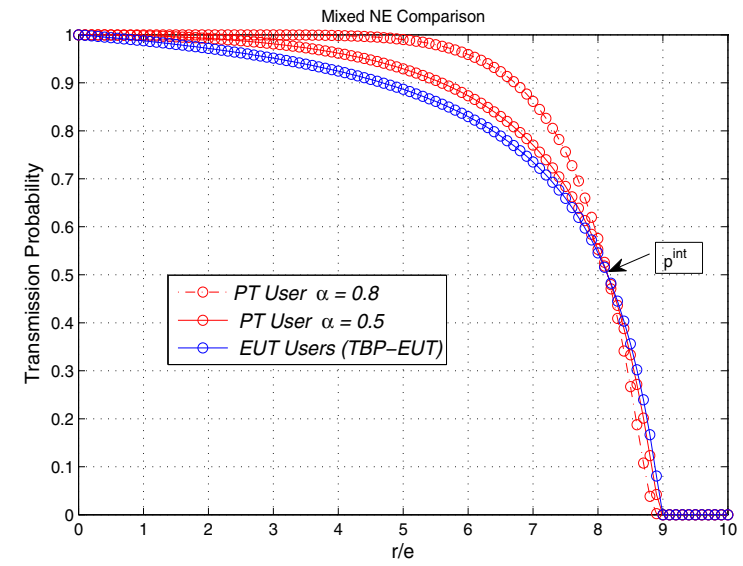

Fig. 16. Transmission probabilities at the operation point for TBP.

$\sum_{i=1,2} r_{i} p_{i} T_{i}=r \sum_{i=1,2} p_{i} T_{i}$. Thus, the service provider will obtain a larger revenue in TBP-EUT. Further, as shown in lemma 5 and Fig. 16, as $r$ increases, the revenue collected will also increase until both TBP-PT and TBP-EUT arrive at the same transmission probability, i.e., $p^{\text {int }}$. Beyond this point, the revenue collected will decrease and TBP-EUT still generates more revenue than TBP-PT.

In the following, we discuss the analytical insights obtained from lemma 22, theorem 23 and theorem 24 with some numerical results. We assume $\alpha_{1}=0.5$ and $\alpha_{2}=0.8$. For both TBPEUT and TBP-PT, two users randomly access the channel with $p_{i \mid\{i\}}=p_{j \mid\{j\}}=0.98, p_{i \mid\{i, j\}}=p_{j \mid\{i, j\}}=0.1, i=1,2$. We begin with a special case where there is no delay penalty for each user as $c_{1}=c_{2}=c=20, e_{1}=e_{2}=e=2, d_{1}=d_{2}=$ $d=0$ and the price charged varies, i.e., $r_{1}=r_{2}=r$. As a result, $v_{i \mid\{t, n t\}}^{t}=v_{j \mid\{t, n t\}}^{t}$ and $v_{i \mid\{t, t\}}^{t}=v_{j \mid\{t, t\}}^{t}$. The system performances are studied and compared as a function of $r / e$.

In Fig. 16, it can be observed that end users' transmission probabilities decrease as the price $r$ increases in both TBPEUT and TBP-PT. Also, in TBP scheme, TBP-PT still suffers a sum average throughput degradation due to PT users' deviation from EUT compared to TBP-EUT as shown in Fig. 17. Moreover, in TBP scheme the service provider's revenue is a linear function of the sum average throughput. Thus, it can be expected that the service provider will obtain a larger revenue in TBP-EUT than TBP-PT for the same price $r$ charged under mild conditions shown in Fig. 18. This is analytically proven in theorem 24.

In conclusion, for the throughput based pricing scheme, we have the following results under mild conditions. First, end users' willingness to use the service will be discouraged by a larger price for both TBP-PT and TBP-EUT. Second, TBPPT suffers a sum average throughput degradation compared to TBP-EUT. As a result, the service provider obtains a larger revenue in TBP-EUT than that in TBP-PT.

Finally, it is interesting to compare two pricing schemes, namely UBP and TBP, for two scenarios (end users are both PT or EUT). In Fig. 19, the revenues the service provider obtains in UBP-PT, UBP-EUT, TBP-PT and TBP-EUT are shown and compared. It can be seen that for all the four

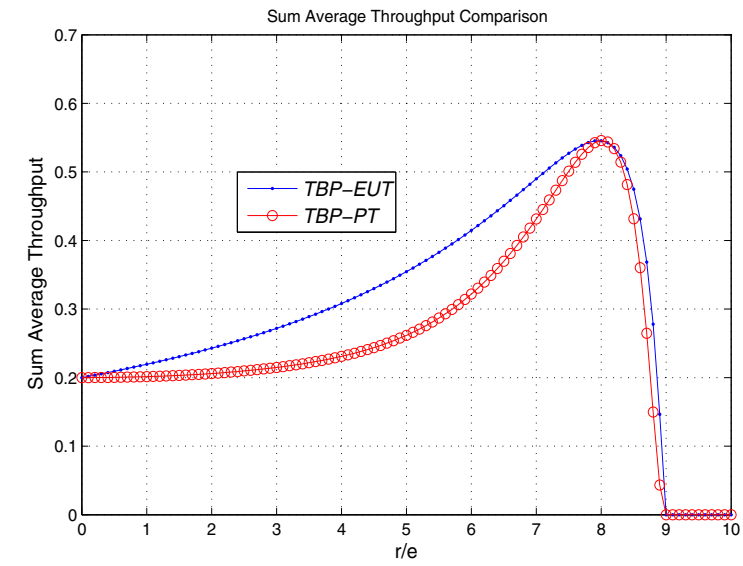

Fig. 17. Sum average throughput at the operation point for TBP.

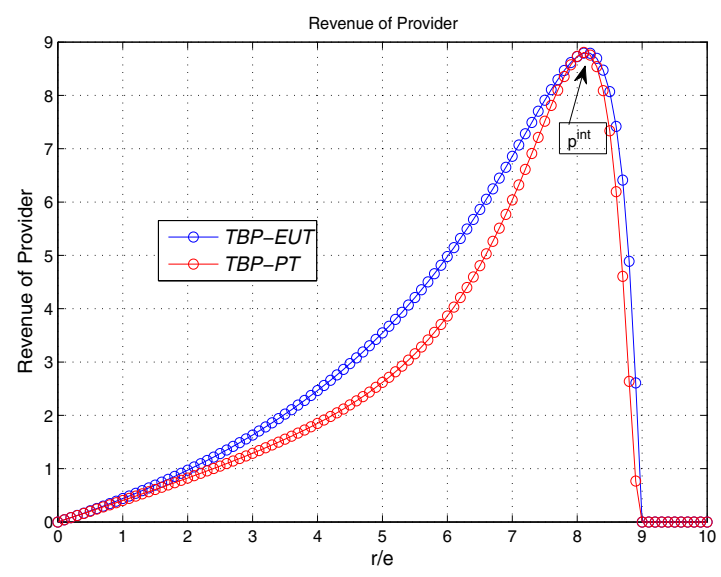

Fig. 18. Service provider's revenue for TBP.

scenarios, the service provider can find an optimal price to achieve the largest revenue. In general, the service provider requires a smaller price to achieve the optimal operating point in the UBP schemes than that in the TBP schemes. The optimal revenues obtained in the four scenarios are similar. In particular, in the TBP schemes, the service provider can achieve the same optimal revenue regardless of the type of the underlying users. This is a useful feature in pricing scheme design since the service provider can employ the same pricing policy without worrying about valuation preference of end users. However, it should be emphasized that there are obvious disparities between the revenues when the optimal price cannot be applied. The service provider should also consider this aspect when designing pricing policies.

\section{CONCLUSION AND Discussion}

Motivated by the increasing amount of end-user control afforded in programmable radio devices, we have envisioned a scenario where end-user actions essentially "interfere" with the underlying engineered system design. In this paper, as an exemplary scenario, we have considered a random access game where players follow the precepts of Prospect Theory (PT), a theory developed by Kahneman and Tversky to explain real-life decision making that often deviates from the 


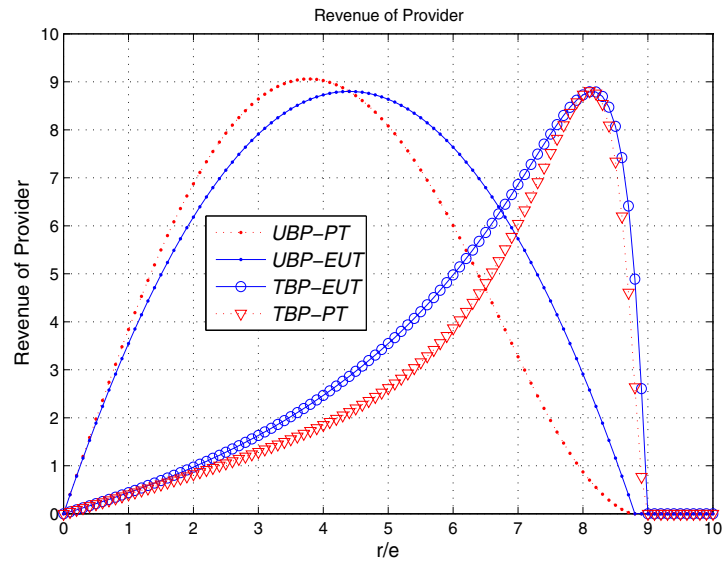

Fig. 19. Service provider's revenue comparison between UBP and TBP.

behavior expected under Expected Utility Theory (EUT). A specific game where selfish players adjust their transmission probabilities over a random access channel under throughput rewards, delay penalties and energy costs has been considered. By analyzing the Nash Equilibrium (NE) achieved in a 2player game, we have proved under mild conditions, that deviations from EUT of any player results in degradation of system throughput and increased delay and energy consumption. We have also studied an $\mathrm{N}$-player homogeneous game with symmetric utility functions and observed similar results at the NE. Finally, we have extended our study to the scenario of data pricing, which is a more practical scenario where end-users' decisions are of paramount importance to the operation of the network. Similar to the wireless random access game, deviation of end users from EUT degrades the system throughput performance under both pricing schemes under consideration (UBP and TBP). Furthermore, it has been shown that end users' deviation from EUT also impacts the service provider's revenues. As a result, it would be a good practice for the service provider to consider the effects of PT in designing pricing schemes.

The results in this paper have only characterized the properties of the mixed NE under both EUT and PT settings. An important aspect of wireless transmission in such networking scenarios is the need for distributed algorithms that can be used by the users in making decisions about transmission probabilities. This future work will necessarily have to rely on an algorithmic game theoretic formulation of the problems considered here or consider heuristic distributed algorithms for updating the transmission probabilities using local information available to the end users. The second important aspect that needs further study is the characterization of the probability weighting functions (different from the parametric weighting function used in this paper) that are specifically pertinent to wireless device usage. This requires psychophysics experiments involving real wireless users and devices and is a topic for future study. A related issue is the design of algorithms when there is uncertainty in characterizing the exact form of the probability weighting function.

\section{REFERENCES}

[1] V. Shah, N. B. Mandayam, and D. J. Goodman, "Power control for wireless data based on utility and pricing," in Proc. 1998 PIMRC, pp. $1427-1432$.

[2] A. B. MacKenzie and S. B. Wicker, "Game theory and the design of selfconfiguring, adaptive wireless networks," IEEE Commun. Mag., vol. 39, pp. 126-131, Nov. 2001.

[3] C. U. Saraydar, N. B. Mandayam, and D. J. Goodman, "Efficient power control via pricing in wireless data networks," IEEE Trans. Commun., vol. 50, no. 2, Feb. 2002.

[4] T. Alpcan, T. Basar, R. Srikant, and E. Altman, "CDMA uplink power control as a noncooperative game," J. Wireless Netw., vol. 8, pp. 659670, Nov. 2002.

[5] P. Liu, M. L. Honig, and S. Jordan, "Forward-link resource allocation for ds-cdma based on pricing," 2000 Wireless Commun. and Networking Conference.

[6] V. Srivastava, J. Neel, A. Mackenzie, R. Menon, L. Dasilva, J. Hicks, J. Reed, and R. Gilles, "Using game theory to analyze wireless ad hoc networks," IEEE Commun. Surveys and Tutorials, vol. 7, no. 4, pp. 4656, Fourth Quarter 2005.

[7] K. Akkarajitsakul, E. Hossain, D. Niyato, and D. I. Kim, "Game theoretic approaches for multiple access in wireless networks: a survey," IEEE Commun. Surveys and Tutorials, vol. 13, no. 3, pp. 372-395, Third Quarter 2011.

[8] J. V. Neumann and O. Morgenstern, Theory of Games and Economic Behavior. Princeton University Press, 1944.

[9] D. Kahneman and A. Tversky, "Prospect theory: an analysis of decision under risk," Econometrica 47, pp. 263-291, 1979.

[10] T. Odean, "Are investors reluctant to realize their losses?" The Journal of Finance, vol. LIII, no. 5, pp. 1775-1798, Oct. 1998.

[11] G. A. Quattrone and A. Tversky, "Contrasting rational and psychological analyses of political choice," The American Political Science Review, vol. 82, no. 3, pp. 719-736, 1988.

[12] C. Camerer, L. Babcock, G. Loewenstein, and R. Thaler, "Labor supply of new york city cab drivers: one day at a time," Quarterly J. Economics, no. 111, pp. 408-441, May 1997.

[13] D. Kahneman and A. Tversky, Choices, Values, and Frames. Cambridge University Press, 2000.

[14] J. Mackie-Mason and H. Varian, "Economic faqs about the internet," in Internet Economics, L. W. McKnight and J. P. Bailey, 1997.

[15] S. M. Choi, E. K. Lee, and M. C. Park, "An empirical study on the determinants of repurchase intention in Korean mobile internet services," 2003 International Telecommunications Society, Asia-Australasian Regional Conference.

[16] T. Li and N. B. Mandayam, "Prospects in a wireless random access game," in Proc. 2012 Conference on Information Sciences and Systems.

[17] J. Mitola, Software Radios: Wireless Architecture for the 21st Century. Wiley, 2000.

[18] _ - "Cognitive radio: an integrated agent architecture for software defined radio," Ph.D. dissertation, Royal Institute of Technology (KTH) Stockholm, Sweden, 2000.

[19] T. Li, N. B. Mandayam, and A. Reznik, "A framework for distributed resource allocation and admission control in a cognitiive digital home," IEEE Trans. Wireless Commun., vol. 12, no. 3, pp. 984-995, Mar. 2013.

[20] T. Li and S. K. Jayaweera, "A novel primary-secondary user power control game for cognitive radios," in Proc. 2008 International Symposium on Information Theory and its Applications.

[21] S. K. Jayaweera and T. Li, "Dynamic spectrum leasing in cognitive radio networks via primary-secondary user power control games," IEEE Trans. Wireless Commun., vol. 8, no. 6, pp. 3300-3310, June 2009.

[22] L. P. Metzger and M. O. Rieger, "Equilibria in games with prospect theory preferences," working paper, Dept. of Banking and Finance, Univeristy of Zurich, Nov. 2009.

[23] D. D. Clark, J. Wroclawski, K. R. Sollins, and R. Braden, "Tussle in cyberspace: defining tomorrow's Internet," IEEE/ACM Trans. Netw., vol. 13, no. 3, pp. 462-475, June 2005.

[24] Y. Wang, A. Nakao, and J. Ma, "Psychological research and application in autonomous networks and systems: a new interesting field," in Proc. 2011 International Conference on Intelligent Computing and Intergrated Systems.

[25] T. Okuda, T. Ichikawa, T. Ideguchi, and X. Tian, "A design method of local community network service systems with ad-hoc network technology," in Proc. 2009 IEEE VTC - Fall.

[26] A. B. MacKenzie and S. B. Wicker, "Selfish users in aloha: a game theoretical approach," in Proc. 2001 IEEE VTC - Fall, vol. 3, pp. 1354-1357. 
[27] E. Altman, R. E. Azouzi, and T. Jimenez, "Slotted aloha as a stochastic game with partial information," Computer Netw., vol. 45, pp. 701-713, Aug. 2004.

[28] T. E. Sagduyu and A. Ephremides, "A game-theoretic analysis of denial of service attacks in wireless random access," Wireless Netw., vol. 15, no. 5, July 2009.

[29] S. Sen, C. Joe-Wong, S. Ha, and M. Chiang, "A survey of broadband data pricing: past proposals, current plans, and future trends," in technical report, Dept. of Electrical Engineering, Princeton University, 2012.

[30] O. Ileri, D. Samardzija, and N. Mandayam, "Demand responsive pricing and competitive spectrum allocation via a spectrum server," in Proc. 2005 IEEE Symp. New Frontiers Dynamic Spectrum Access Networks, pp. 194-202.

[31] O. Ileri and N. B. Mandayam, "Dynamic spectrum access models: toward an engineering perspective in the spectrum debate," in IEEE Commun. Mag., pp. 153-160, Jan. 2008.

[32] P. Marbach and R. Berry, "Downlink resource allocation and pricing for wireless networks," in Proc. 2002 IEEE INFOCOM.

[33] A. Tversky and D. Kahneman, "Advances in prospect theory: cumulative representation of uncertainty," J. Risk and Uncertainty, vol. 5, pp. 297323, 1992.

[34] D. Prelec, "The probability weighting function," Econometrica, pp. 497528, 1998.

[35] M. J. Osborne and A. Rubinstein, A Course in Game Theory. The MIT Press, 1994

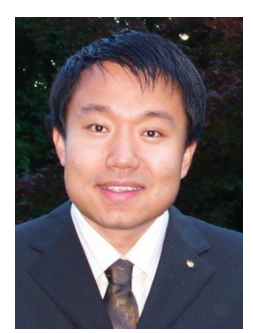

Tianming Li received his B.E. degree from Shanghai Jiao Tong University, Shanghai, China in 2006 and M.S. degree from University of New Mexico, Albuquerque, USA in 2008, both in Electrical Engineering. In October 2013, he obtained his Ph.D. degree from WINLAB, Electrical and Computer Engineering Department, Rutgers University. His research interests include efficient resource allocation, management and data pricing schemes of communication networks. Moreover, he has collaborated with Dr. Mandayam to study the end-user effects on wireless networks by using mathematical modeling and economic theories and models.

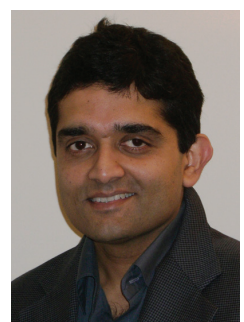

Narayan B. Mandayam is currently the Peter D. Cherasia Faculty Scholar at Rutgers University. He received the B.Tech (Hons.) degree in 1989 from the Indian Institute of Technology, Kharagpur, and the M.S. and Ph.D. degrees in 1991 and 1994 from Rice University, all in electrical engineering. From 1994 to 1996, he was a Research Associate at the Wireless Information Network Laboratory (WINLAB), Rutgers University before joining the faculty of the Electrical and Computer Engineering department at Rutgers where he became Associate Professor in 2001 and Professor in 2003. Currently, he also serves as Associate Director at WINLAB. He was a visiting faculty fellow in the Department of Electrical Engineering, Princeton University in 2002 and a visiting faculty at the Indian Institute of Science in 2003. Dr. Mandayam's research interests are in various aspects of wireless data transmission with emphasis on techniques for cognitive radio networks including their implications for spectrum policy. Using constructs from game theory, communications and networking, his work focusses on radio resource management as well as signal processing for enabling wireless technologies to support various applications.

Dr. Mandayam is a co-recipient of the 2014 IEEE Donald G. Fink Award for his IEEE PROCEEDINGS paper titled "Frontiers of Wireless and Mobile Communications," and the 2009 Fred W. Ellersick Prize from the IEEE Communications Society for his work on dynamic spectrum access models and spectrum policy. He is also a recipient of the Institute Silver Medal from the Indian Institute of Technology in 1989 and the National Science Foundation CAREER Award in 1998. He is a coauthor of the books: Principles of Cognitive Radio (Cambridge University Press, 2012), and Wireless Networks: Multiuser Detection in Cross-Layer Design (Springer, 2004). He has served as an Editor for the journals IEEE COMMUNICATION LETTERS and IEEE TRANSACTIONS ON WIRELESS COMMUNICATIONS. $\mathrm{He}$ has also served as a guest editor of the IEEE JSAC Special Issues on Adaptive, Spectrum Agile and Cognitive Radio Networks (2007), and Game Theory in Communication Systems (2008). He is a Fellow of the IEEE and currently serves as a Distinguished Lecturer of the IEEE. 